TRANSACTIONS OF THE

AMERICAN MATHEMATICAL SOCIETY

Volume 353, Number 12, Pages 4687-4727

S 0002-9947(01)02807-0

Article electronically published on June 27, 2001

\title{
ORTHOGONAL, SYMPLECTIC AND UNITARY REPRESENTATIONS OF FINITE GROUPS
}

\author{
CARL R. RIEHM
}

\begin{abstract}
Let $K$ be a field, $G$ a finite group, and $\rho: G \rightarrow \mathbf{G L}(V)$ a linear representation on the finite dimensional $K$-space $V$. The principal problems considered are:

I. Determine (up to equivalence) the nonsingular symmetric, skew symmetric and Hermitian forms $h: V \times V \rightarrow K$ which are $G$-invariant.

II. If $h$ is such a form, enumerate the equivalence classes of representations of $G$ into the corresponding group (orthogonal, symplectic or unitary group).

III. Determine conditions on $G$ or $K$ under which two orthogonal, symplectic or unitary representations of $G$ are equivalent if and only if they are equivalent as linear representations and their underlying forms are "isotypically" equivalent.

This last condition means that the restrictions of the forms to each pair of corresponding isotypic (homogeneous) $K G$-module components of their spaces are equivalent.

We assume throughout that the characteristic of $K$ does not divide $2|G|$.

Solutions to I and II are given when $K$ is a finite or local field, or when $K$ is a global field and the representation is "split". The results for III are strongest when the degrees of the absolutely irreducible representations of $G$ are odd - for example if $G$ has odd order or is an Abelian group, or more generally has a normal Abelian subgroup of odd index - and, in the case that $K$ is a local or global field, when the representations are split.
\end{abstract}

Let $G$ be a finite group of order $g, K$ a field of characteristic relatively prime to $2 g$ with an involution (possibly the identity) $a \mapsto \bar{a}$, and $\rho: G \rightarrow \mathbf{G L}(V)$ a linear representation of $G$ on the finite dimensional $K$-vector space $V$.

The group algebra $K G$ has an involution ${ }^{-}$extending that on $K$ and inverting the group elements. Since $K G$ is a semisimple algebra, the involution algebra $\left(K G,^{-}\right)$ is a direct sum of simple involution algebras:

$$
\left(K G,^{-}\right)=\left(A_{1},{ }^{-}\right) \oplus\left(A_{2},{ }^{-}\right) \oplus \cdots \oplus\left(A_{r},{ }^{-}\right),
$$

where each $A_{i}$ either is a simple algebra stabilized by the involution or is a "simple hyperbolic algebra" - a direct sum of two simple algebras interchanged by the involution. Now let

$$
h: V \times V \rightarrow K
$$

Received by the editors April 5, 2000 and, in revised form, October 30, 2000.

2000 Mathematics Subject Classification. Primary 20C99, 11E08, 11E12; Secondary 20G05, $51 \mathrm{~F} 25$. 
be a symmetric, skew symmetric or Hermitian form on $V$ (we assume throughout that all forms are nonsingular) which is $G$-invariant. The decomposition

$$
V=A_{1} V \oplus A_{2} V \oplus \cdots \oplus A_{r} V
$$

of the $K G$-module $V$ into "isotypic" components is an orthogonal direct sum. $\left(A_{i} V\right.$ is the direct sum of two isotypic components in the usual sense if $A_{i}$ is hyperbolic.) The restrictions of $h$ to these isotypic components are called the isotypic components of $h$, and two forms are called isotypically equivalent if their corresponding isotypic components are equivalent.

To say that $h$ is $G$-invariant is the same as saying that $\rho$ is an orthogonal representation $G \rightarrow \mathbf{O}(V, h)$, a symplectic representation $G \rightarrow \mathbf{S p}(V, h)$ or a unitary representation $G \rightarrow \mathbf{U}(V, h)$, according to the type of $h$. We refer to such representations as equivariant representations.

Two equivariant representations $\rho$ and $\rho^{\prime}$ are (equivariantly) equivalent if there is an isometry between their forms which is also $K G$-linear. There are therefore two obvious necessary conditions for $\rho$ and $\rho^{\prime}$ to be equivalent, namely

(i) $\rho$ and $\rho^{\prime}$ are equivalent as linear representations, and

(ii) their underlying forms are isotypically equivalent.

This allows us to restrict our attention by and large to the isotypic case, that is to say, when $V=A_{i} V$ for some $i$. (In this case we say that $V$, or the associated representation of $G$, is $A_{i}$-isotypic or isotypic of type $A_{i}$.) Furthermore if the involution algebra $\left(A_{i},{ }^{-}\right)$is hyperbolic, linear equivalence implies equivariant equivalence, and so we can assume in addition that $A_{i}$ is a simple algebra.

Problems I, II and III were solved earlier for $K$ algebraically closed or real closed.

In the algebraically closed case, Frobenius showed that two orthogonal or two symplectic representations are equivalent if and only if they are equivalent as linear representations (13, pp. 184-186); furthermore, Frobenius and Schur characterized linear representations which are equivalent to an orthogonal or symplectic representation (cf. [22], §13.2), that is to say, for which there is a $G$-invariant symmetric or skew symmetric form.

In the case of a real closed field $K$, Fröhlich and McEvett showed (9], Prop. 4.9 ) that two unitary (over $K(\sqrt{-1})$ ) or orthogonal representations are equivalent if and only if they are linearly equivalent and their underlying forms are isotypically equivalent. They also showed that an invariant symmetric or Hermitian form on an irreducible $K G$-module is definite. Of course the existence of an invariant positive definite symmetric or Hermitian form on an arbitrary $K G$-module $V$ is well known when $K$ is formally real - one such form is $\sum_{s \in G} f(s x, s y)$, where $f$ is any positive definite symmetric or Hermitian form on $V$.

Recently, G. Nebe has given a method in 16 for calculating, under certain circumstances, the determinant or the Hasse invariant of an invariant symmetric form of a representation of a perfect group over a totally real number field, and in [17] she gives a recursion formula for the invariant symmetric form of certain irreducible representations of a symmetric group. This form is also determined, using different methods, in [10] - we give an example in $\$ 6]$

Another result relevant here is the following (cf. \$1.3): If $\rho: G \rightarrow \mathbf{G L}(V)$ is a given linear representation, there is at least one $G$-invariant form $h$ of a given type (symmetric, skew symmetric or Hermitian) on $V$ if and only if the following hold: 
1. In the symmetric case, $\operatorname{len}_{A} A V$ is even if $\left(A,^{-}\right)$is a simple symplectic component of $\left(K G,{ }^{-}\right)$of index 1.

2. In the skew symmetric case, $\operatorname{len}_{A} A V$ is even if $\left(A,,^{-}\right)$is a simple orthogonal component of $\left(K G,^{-}\right)$of index 1.

3. $\operatorname{dim}_{K} A V=\operatorname{dim}_{K} \bar{A} V$ for all simple algebra components $A$ of $K G$.

This is an easy consequence of results of McEvett [14].

Before summarizing our results, it is necessary to introduce some additional notions and notation. Unexplained terms can be found in $\$ 1$

Let $A$ be a simple $K$-algebra, say $A \cong \mathrm{M}(n, D)$ for the division algebra $D$ with center $L$. If ${ }^{-}$is an involution on $A$ stabilizing $K, K_{0}$ denotes the subfield of elements of $K$ fixed by the involution, and $L_{0}$ that of $L$.

An arbitrary linear representation is called split if it is the sum of absolutely irreducible representations. It is said to be of odd type if $A V=0$ for each simple summand $A$ of $K G$ which has even degree and which is stable under the canonical involution. We shall say that $G$ has odd representation type (with respect to $K$ and its involution) if all nonhyperbolic simple involution components of $K G$ are quasisplit and of odd degree. This is the case, for example, if $G$ has odd order.

In general, we ignore the case of a unitary representation with respect to a skew Hermitian form, since any such form is a scalar multiple of an Hermitian form.

Since skew symmetric forms are equivalent if and only if they have the same rank, symplectic representations are particularly easy to deal with. In particular since a space $V$ has at most one equivalence class of skew symmetric forms, invariant or not, problem $\mathbf{I}$ is trivial.

\section{Symplectic representations (cf. \$2).}

II Let $A$ be a simple algebra component of $K G$, stable under ${ }^{-}$, and let $m$ be a positive integer.

$\left(A,{ }^{-}\right)$orthogonal. Suppose that $A$ has index 1 or that $K$ is finite, real closed, local or global. Then there is exactly one equivalence class of $A$ isotypic symplectic representations $G \rightarrow \mathbf{S p}(V)$ of length $m$ (assuming that there is at least one such symplectic representation - i.e. that $m$ is even if the index of $A$ is 1$)$.

$\left(A,{ }^{-}\right)$symplectic. The equivalence classes of A-isotypic symplectic representations $G \rightarrow \mathbf{S p}(V)$ of length $m$ are in bijective correspondence with the equivalence classes of symmetric bilinear forms over $L$ (respectively skew Hermitian forms over $D$ ) of rank $m$, if $A$ has index 1 (respectively $>1$ ).

It follows that the number of equivalence classes of such symplectic representations $G \rightarrow \mathbf{S p}(V)$ is

$$
\begin{array}{ll}
2 & \text { if } K \text { is finite, } \\
\left(L^{*}: L^{* 2}\right) \text { resp. } 2\left(L^{*}: L^{* 2}\right) & \text { if } K \text { is local, } A \text { has index } 1, \text { and } \\
& m=1 \text { respectively } m>1, \\
\left(L^{*}: L^{* 2}\right)-\delta_{1 m} & \text { if } K \text { is local and } A \text { has index }>1, \\
1 & \text { if } K \text { is real closed, } \\
\infty & \text { if } K \text { is global. }
\end{array}
$$

$\left(A,{ }^{-}\right)$unitary. The equivalence classes of $A$-isotypic symplectic representations $G \rightarrow \mathbf{S p}(V)$ of length $m$ are in bijective correspondence with the skew Hermitian forms of rank $m$ over $D$. 
Thus the number of equivalence classes of such symplectic representations $G \rightarrow \mathbf{S p}(V)$ is

$$
\begin{array}{ll}
1 & \text { if } K \text { is finite, } \\
2 & \text { if } K \text { is local, } \\
m+1 & \text { if } K \text { is real closed, } \\
\infty & \text { if } K \text { is global. }
\end{array}
$$

III Let $\rho$ and $\rho^{\prime}$ be symplectic representations of $G$. Suppose that at least one of the following conditions holds:

1. $G$ is a symmetric group.

2. $K$ is finite.

3. $\rho$ is split.

4. If $\left(A,{ }^{-}\right)$is a unitary simple involution component of $K G$ such that $A V \neq 0$ under the action provided by $\rho$, then $\left(A,{ }^{-}\right)$is hyperbolic.

Assume in addition that $\rho$ is of odd type in cases 2, 3 and 4; then $\rho$ and $\rho^{\prime}$ are equivalent if and only if they are linearly equivalent.

From this we get a conjugacy result:

Let $G$ and $G^{\prime}$ two subgroups of $\mathbf{S p}(V, h)$ of order relatively prime to char $K$ such that either

1. $G$ is a symmetric group, or

2. $G$ and $G^{\prime}$ are of odd representation type, and $K$ is either finite or a splitting field for $G$.

Then $G$ and $G^{\prime}$ are conjugate in $\mathbf{S p}(V, h)$ if and only if they are conjugate in $\mathbf{G L}(V)$.

For the determinant $\operatorname{det}\left({ }^{-}\right)$of an involution, see $\$ \underline{1.2}$.

Unitary representations (cf. Theorem 3.1, Theorem 4.1, Corollary 4.4, Theorem 5.2).

\section{K finite.}

I, II and III. There is only one equivalence class of Hermitian forms of a given rank, invariant or not, and two arbitrary (not necessarily isotypic) unitary representations of $G$ are equivalent if and only if they are linearly equivalent.

\section{K local.}

I and II. Let $A$ be a simple algebra component of $K G$, stable under ${ }^{-}, V$ an $A$-module of length $m$, and $h$ an Hermitian form on $V$. There is a $G$-invariant Hermitian form on $V$ equivalent to $h$ if and only if $n$ is odd or $\operatorname{det} h=\mathrm{N}_{L / K}\left(\operatorname{det}\left({ }^{-}\right)\right)^{m}$. If $h^{\prime}$ is an invariant form, there is exactly one class of unitary representations $G \rightarrow \mathbf{U}\left(V, h^{\prime}\right)$ if $n$ is odd; if $n$ is even, there are two.

III. Two unitary representations of $G$ of odd type are equivalent if and only if they are equivalent as linear representations and their underlying forms are isotypically equivalent.

$\mathbf{K}$ global. Assume that $A$ is a simple component of $K G$ which is split and is stable under ${ }^{-}$. Let $V$ be an $A$-module of length $m$ and $h$ an Hermitian form on $V$. Let $\wp$ be the set of real primes of $K_{0}$ which do not split in $K$.

I. There is an invariant form on $V$ equivalent to $h$ if and only if the positive and negative indices of $h$ are divisible by $n$ at each $\mathfrak{p} \in \wp$ and, when $n$ is even, $\operatorname{det} h=\left(\operatorname{det}\left({ }^{-}\right)\right)^{m}$. 
II. Let $h^{\prime}$ be an invariant form on $V$.

(i) If $n$ is odd, there is exactly one equivalence class of unitary representations $G \rightarrow \mathbf{U}\left(V, h^{\prime}\right)$ for which $V$ is A-isotypic.

(ii) If $n$ is even, the equivalence classes of unitary representations $G \rightarrow \mathbf{U}\left(V, h^{\prime}\right)$ for which $V$ is A-isotypic are in bijective correspondence with those norm classes in $K_{0}^{*} / \mathrm{N}_{K / K_{0}} K^{*}$ which are positive at each $\mathfrak{p} \in \wp$.

III. Two split unitary representations of $G$ of odd type are equivalent if and only if they are equivalent as linear representations and their forms are isotypically equivalent.

Witt equivalence between symmetric forms is denoted by $h \sim_{W} g$.

Orthogonal representations (cf. Theorem 3.1, Theorem4.1, Corollary 4.4, Theorem 4.9. Theorem [5.3). Let $A$ be a simple algebra component of $K G$, stable under ${ }^{-}$, and let $m$ be a positive integer.

K finite.

I, II. Let $V$ be an $A$-isotypic module of length $m$.

$\left(A,{ }^{-}\right)$symplectic. $V$ supports an invariant symmetric form if and only if $m$ is even. If $V$ does have an invariant symmetric form, every invariant form is hyperbolic and there is exactly one equivalence class $G \rightarrow \mathbf{O}(V)$ of A-isotypic orthogonal representations.

$\left(A,{ }^{-}\right)$orthogonal. When $n$ is odd, there are invariant forms of both determinants on $V$ and there is exactly one equivalence class of orthogonal representations for each of these forms.

When $n$ is even, an invariant form $h$ on $V$ has determinant

$$
\mathrm{N}_{L / K}\left(\operatorname{det}\left({ }^{-}\right)\right)^{m}
$$

and there are two equivalence classes of orthogonal representations $G \rightarrow$ $\mathbf{O}(V, h)$.

$\left(A,{ }^{-}\right)$unitary. An invariant form on $V$ has determinant

$$
\left(-\operatorname{disc}\left(L / L_{0}\right)\right)^{m n\left|L_{0}^{*}\right| /\left|K^{*}\right|}
$$

and supports exactly one equivalence class of orthogonal representations. III. Let $\rho: G \rightarrow \mathbf{O}(V, h)$ be an orthogonal representation such that $B V=0$ for every component $\left(B,^{-}\right)$of $\left(K G,^{-}\right)$which is orthogonal and of even degree. If $\rho^{\prime}$ is another orthogonal representation on $V$, then $\rho$ and $\rho^{\prime}$ are equivalent if and only if they are linearly equivalent and their forms are isotypically equivalent. In particular, if $G$ has odd representation type, two orthogonal representations are equivalent if and only if they are linearly equivalent and their forms are isotypically equivalent.

K local. I, II. See Theorems 4.1 and 4.9

III. Let $\rho$ and $\rho^{\prime}$ be orthogonal representations of $G$ of odd type, and assume that either $\rho$ is split or $G$ is Abelian. Then $\rho$ and $\rho^{\prime}$ are equivalent if and only if they are linearly equivalent and their forms are isotypically equivalent.

$\mathbf{K}$ global. Assume that $A$ is split.

$\left(A,{ }^{-}\right)$symplectic.

I, II. If $h$ is an invariant symmetric form on the A-isotypic module $V$, it is hyperbolic and the orthogonal representation it affords is unique up to equivalence. 
$\left(A,{ }^{-}\right)$orthogonal.

I. See Theorem 5.3 .

II. If $h$ is an invariant symmetric form on the A-isotypic module $V$, there is (up to equivalence) only one orthogonal representation $G \rightarrow$ $\mathbf{O}(V, h)$ if $n$ is odd, and there are an infinite number of equivalence classes of orthogonal representations $G \rightarrow \mathbf{O}(V, h)$ if $n$ is even.

III. Two split orthogonal representations of odd type are equivalent if and only if they are equivalent as linear representations and their underlying forms are isotypically equivalent.

The case $K$ local, $\left(A,^{-}\right)$orthogonal, $D=L \neq K, n$ odd, and $m=1$ is not completely resolved; its solution depends on the calculation of "trace forms" in the extension $L / K$. This is carried out in Theorem 4.9 when $K$ is nondyadic (which means, given our assumptions on $K$, that $K$ is not a finite extension of the 2-adic numbers $\mathbf{Q}_{2}$ ). The dyadic case is unresolved, but a partial solution (for the trace form problem) can be found in [6].

The proofs, when $V=A V$ with $A$ simple, are based on the determination of $h$ in terms of the matrix $h_{0}$ (cf. \$1.2) and the discriminant matrix $\underline{\breve{h}}$ of the form $\breve{h}$ over $D$ which corresponds to $\rho$ under Hermitian Morita equivalence (cf. \$1.4). This computation appears in its simplest form when $A$ is split: then the discriminant matrix of $h$ is simply $\frac{n}{g}\left(\epsilon_{0} \underline{\breve{h}} \otimes h_{0}^{t}\right)$, where $h_{0}^{*}=\epsilon_{0} h_{0}$.

There is an extensive theory calculating the Witt group of equivariant representations, even in the integral case - cf. [23, 4]. But this work does not concern itself with the questions dealt with in this paper.

If $K$ is a global field, we give a proof of the Hasse Principle (Theorem [5.6) for equivariant representations of finite groups over $K$. It describes the precise conditions under which the equivalence of two equivariant representations over a global field $K$ is implied by their equivalence over all completions of $K$.

This theorem is well-known in the sense that anyone familiar with the FröhlichMcEvett theory [9] and the Hasse Principle for $\epsilon$-Hermitian forms over division algebras over global fields (cf. 21], p. 347) is aware that such a result exists. But to my knowledge, it is not documented in the literature.

The Hasse Principle always holds for unitary representations, but its validity in the orthogonal and symplectic cases depends on the nature of the representations as linear representations - see Corollary [5.10,

\section{Preliminaries}

1.1. Sesquilinear forms over fields and division algebras. If $h: V \times V \rightarrow D$ is a form over $D$, we denote by $\operatorname{det} h \in L^{*} / \mathrm{N}_{L / L_{0}} L^{*}$ the "norm class" of the reduced norm $\operatorname{nrd}_{B / L} \underline{h}$, where $\underline{h} \in B=\mathrm{M}(k, D)$ is the matrix of $h$ with respect to some basis of $V$ over $D$ and $k=\operatorname{dim}_{D} V$. We interpret $\mathrm{N}_{L / L_{0}} L^{*}$ to be $=L^{* 2}$ if the involution is the identity on $L$, i.e. if the involution on $D$ is of the first kind. We note that $\operatorname{det} h \in L_{0}^{*} / \mathrm{N}_{L / L_{0}} L^{*}$ if $h$ is Hermitian.

We also define the discriminant of a symmetric bilinear form $h$ (when $D=L$ ) to be $\operatorname{disc} h=(-1)^{k(k-1) / 2} \operatorname{det} h$, and that of a skew Hermitian form over $L$ to be $\operatorname{disc} h=\operatorname{det} \sqrt{\lambda_{0}} h \in L_{0}^{*} / \mathrm{N}_{L / L_{0}} L^{*}$, where $L=L_{0}\left(\sqrt{\lambda_{0}}\right)$. Of course $\sqrt{\lambda_{0}} h$ is an Hermitian form. We put $\operatorname{disc} h=\operatorname{det} h$ if $h$ is Hermitian.

Lemma 1.1. Let $L / K$ be a finite separable extension such that if the involution on $L$ is $\neq$ the identity, then it is also $\neq$ the identity on $K$. Suppose that $h: V \times V \rightarrow L$ 
is either a nonsingular symmetric bilinear form or a nonsingular Hermitian or skew Hermitian form of rank $k$. Then the transfer $\operatorname{Tr}_{L / K} h: V \times V \rightarrow K$ has determinant

$$
\operatorname{det} \operatorname{Tr}_{L / K} h=(\operatorname{disc}(L / K))^{k} \mathrm{~N}_{L / K}(\operatorname{det} h),
$$

where $\operatorname{disc}(L / K)$ is the field discriminant. Similarly,

$$
\operatorname{disc} \operatorname{Tr}_{L / K} h=\left((-1)^{l(l-1) / 2} \operatorname{disc}(L / K)\right)^{k} \mathrm{~N}_{L / K}(\operatorname{disc} h),
$$

where $l=(L: K)$. If $h$ is Hermitian, we also have

$$
\operatorname{det} \operatorname{Tr}_{L / K} h=\left(\operatorname{disc}\left(L_{0} / K_{0}\right)\right)^{k} \mathrm{~N}_{L_{0} / K_{0}}(\operatorname{det} h) .
$$

A proof for the bilinear case can be found, e.g., in [15], Lemma 2.2, and a similar proof applies to the Hermitian and skew Hermitian cases if one carries out the calculation using a basis of $L_{0}$ over $K_{0}$.

The following result of Milnor ([15], pp. 89 and 91) is critical in the study of equivariant representations over local fields:

Lemma 1.2. If $L / K$ is a finite separable extension of local fields, the transfers from $L$ to $K$ of two inequivalent nonsingular symmetric forms with the same determinant are inequivalent over $K$. Similarly, if $L$ has a nonidentity involution which is the identity on $K$, the transfers of two inequivalent Hermitian forms from $L$ to $K$ remain inequivalent, in fact have the same determinant but different Hasse symbols.

Let $b=\left\langle a_{1}, \ldots, a_{m}\right\rangle$ be a diagonalization of the symmetric bilinear form $b$. We use the definition $\mathrm{S}_{K}(b)=\mathrm{S}(b)=\prod_{i<j}\left(a_{i}, a_{j}\right)_{K}$ of the Hasse-Witt invariant, where $\left(a_{i}, a_{j}\right)_{K}$ stands for the Brauer class of the quaternion algebra - which we also denote by $\left(a_{i}, a_{j}\right)_{K}$.

Lemma 1.3. (i) If $\eta \in K^{*}$,

$$
\mathrm{S}(\eta b)=\mathrm{S}(b)(\eta,-1)_{K}^{m(m-1) / 2}(\eta, \operatorname{det} b)_{K}^{m-1} .
$$

(ii) If $b_{1}, \ldots, b_{r}$ are symmetric forms over $K$,

$$
\mathrm{S}\left(b_{1} \perp \ldots \perp b_{r}\right)=\prod_{i} \mathrm{~S}\left(b_{i}\right) \prod_{i<j}\left(\operatorname{det} b_{i}, \operatorname{det} b_{j}\right)_{K} .
$$

(iii) If $\eta_{1}, \ldots, \eta_{n} \in K^{*}$,

$$
\begin{aligned}
& \mathrm{S}\left(\left\langle\operatorname{det} \eta_{1} b, \ldots, \operatorname{det} \eta_{n} b\right\rangle\right) \\
& \quad=\mathrm{S}\left(\left\langle\eta_{1}^{m}, \ldots, \eta_{n}^{m}\right\rangle\right)(\operatorname{det} b,-1)_{K}^{n(n-1) / 2}\left(\operatorname{det} b, \eta_{1} \cdots \eta_{n}\right)_{K}^{m(n-1)} .
\end{aligned}
$$

(iv) Let $h_{0}$ be another symmetric form, say of rank $n$. Then

$\mathrm{S}\left(b \otimes h_{0}\right)=\mathrm{S}(b)^{n} \mathrm{~S}\left(h_{0}\right)^{m}(\operatorname{det} b,-1)_{K}^{n(n-1) / 2}\left(\operatorname{det} b, \operatorname{det} h_{0}\right)_{K}^{m n-1}\left(\operatorname{det} h_{0},-1\right)_{K}^{m(m-1) / 2}$.

(v) If $b$ is hyperbolic of dimension $m=2 k$, then

$$
\mathrm{S}(b)=(-1,-1)_{K}^{k(k-1) / 2} .
$$

These are either well known or easily checked.

The equivalence theory of forms over finite, real closed, local (non-Archimedean) and global fields is summarized in the following table. It is derived from [21], p. 347. In it, "1 $1^{\text {st }}$ k." refers to the involution being of the first kind (cf. \$1.2), and "Hasse" means that the Hasse principle holds. 


\begin{tabular}{|l||c|c|c|c|}
\hline Type of form & finite & real & local & global \\
\hline \hline symmetric & $\operatorname{dim}, \operatorname{det}$ & $\operatorname{dim}, \operatorname{sgn}$ & $\operatorname{dim}, \operatorname{det}, \mathrm{S}$ & Hasse \\
\hline skew-symmetric & $\operatorname{dim}$ & $\operatorname{dim}$ & $\operatorname{dim}$ & $\operatorname{dim}$ \\
\hline Herm/quat.(1 $\left.1^{\text {st }} \mathrm{k}.\right)$ & $\nexists$ & $\operatorname{dim}, \mathrm{sgn}$ & $\operatorname{dim}$ & Hasse \\
\hline skew-Herm/quat. & $\nexists$ & $\operatorname{dim}$ & $\operatorname{dim}, \operatorname{det}$ & \\
\hline Herm/field & $\operatorname{dim}$ & $\operatorname{dim}, \operatorname{sgn}$ & $\operatorname{dim}, \operatorname{det}$ & $\operatorname{dim}, \operatorname{det}, \operatorname{sgn}_{\mathfrak{p}}$ \\
\hline Herm/quat.($\left(2^{\text {nd }} \mathrm{k}.\right)$ & $\nexists$ & $\nexists$ & $\nexists$ & $\operatorname{dim}, \operatorname{det}, \operatorname{sgn}_{\mathfrak{p}}$ \\
\hline
\end{tabular}

TABLE 1.

\subsection{Simple involution algebras and representations of finite groups.}

Lemma 1.4. The center $L$ of any simple direct summand $A$ of $K G$ is Abelian over $K$. And if $A$ is stable under the canonical involution ${ }^{-}$of $K G, L / K_{0}$ is also Abelian.

Proof. If $K$ is a finite field, $L / K$ is of course Abelian, and the same is true for an arbitrary field $K$ of characteristic $\neq 0$ by extension of scalars from the finite case. If the characteristic of $K$ is 0 , it is well known that $L$ is a subfield of a cyclotomic extension of $K$ (cf. [3], $\S 70$ ) - in fact it is generated by the character values of any absolutely irreducible representation $\rho$ arising from $A$ - and so is Abelian.

Suppose then that $A$ is stable under the involution of $K G$ and that $K \neq K_{0}$. Let $A_{1}$ be the simple direct summand of $K_{0} G$ from which $A$ arises by extension of scalars. If $L_{1}$ is the center of $A_{1}, K \otimes_{K_{0}} L_{1}$ is the center of $K \otimes_{K_{0}} A_{1} \subset K G$. By [1], Prop. 3, $K \otimes_{K_{0}} L_{1}$ is the direct sum of the composites of $K$ and $L_{1}$ over $K_{0}$, and therefore either $K \otimes_{K_{0}} L_{1}$ is a field, namely $L$, or $K \otimes_{K_{0}} L_{1} \cong L_{1} \oplus L_{1}$. In the first case, the group generated by the automorphisms ${ }^{-} \otimes \mathrm{id}_{L_{1}}$ and $\mathrm{id}_{K} \otimes \sigma$ $\left(\sigma \in \operatorname{Gal}\left(L_{1} / K_{0}\right)\right)$ is Abelian and has order $2\left[L_{1}: K_{0}\right]$ and so is the Galois group of $L / K_{0}$. In the second case $L_{1}=L$.

By Brauer's Theorem ([22, p. 24), every representation of $G$ over $K$ is split (i.e. $K$ is a splitting field of $G$ ) if the exponent of $G$ divides the order $|\mu(K)|$ of the group of roots of unity of $K$, or if it divides the order of the multiplicative group of the residue class field when $K$ is local.

We note that $G$ has odd representation type if it has odd order or is Abelian, or more generally if it has a normal Abelian subgroup of odd index. See [22], Corollary, p. 61, for the case of characteristic 0. The case of prime characteristic $p$ follows from the fact that any absolutely irreducible representation over a field of characteristic $p$ which contains a primitive $g^{\text {th }}$ root of unity is the reduction "mod $p$ " of an absolutely irreducible representation in characteristic 0 .

The algebra $A \cong \mathrm{M}(n, D)$ is called split if $D=K$, quasisplit or of (Schur) index 1 if $D=L$. Otherwise $A$ is "not quasisplit" or it is "of index $>1$ ". The index of $A$ is $d$ and the degree of $A$ is $n d$, where $\operatorname{dim}_{L} D=d^{2}$.

An involution on the simple algebra $A$ is of the first kind if the involution is the identity on the center of $A$; otherwise it is of the second kind, or unitary. An involution of the first kind is orthogonal if its 1-eigenspace has dimension $\frac{1}{2} n d(n d+1)$ (where $n d$ is the degree of $A$ ) over the center of $A$; otherwise it is symplectic - in which case the 1-eigenspace has dimension $\frac{1}{2} n d(n d-1)$. 
A hyperbolic involution component of $K G$ is also considered to be of the second kind.

If the simple algebra $A \cong \mathrm{M}(n, D)$ has an involution, then the division algebra $D$ has one of the same kind. If $D$ is not a field, it can be chosen to be a symplectic involution; in this case we denote it by * .

If $D$ is a quaternion algebra over $K,{ }^{*}$ will be assumed to be the standard conjugation on $D$. In this case $D_{0}$ denotes the skew conjugate elements, the "pure" quaternions. If ${ }^{-}$is an orthogonal involution on $D, \bar{d}=j^{-1} d^{*} j$ for $j \in D_{0}$ uniquely determined up to a nonzero element of $K$.

Note that if $K$ is a local field, a quaternion division algebra $D$ does not admit an involution of the second kind ([21], Theorem 2.2 (ii), p. 353).

The symbol $a^{*}$ will also denote the "transpose conjugate" of a matrix $a \in$ $\mathrm{M}(n, D)$, or more generally of a rectangular matrix with entries in $D$. If $A$ is identified with $\mathrm{M}(n, D)$, there is a matrix $h_{0} \in A$ such that $\bar{a}=h_{0}^{-1} a^{*} h_{0}$, satisfying $h_{0}^{*}=\epsilon_{0} h_{0}$ for $\epsilon_{0}= \pm 1$; we can and shall suppose that $\epsilon_{0}=1$ if the involution on $A$ is unitary.

Suppose that the involution ${ }^{-}$on $A$ is of the first kind. If $D=L$, the transpose on $A$ is an orthogonal involution, and ${ }^{-}$is an orthogonal respectively symplectic involution if $h_{0}$ is a symmetric respectively skew symmetric matrix. If $D$ is a quaternion algebra, then transpose conjugate is a symplectic involution, and the involution - on $A$ is a symplectic respectively orthogonal involution if $h_{0}$ is an Hermitian respectively skew Hermitian matrix (with respect to ${ }^{*}$ ).

If the involution ${ }^{-}$on $A$ is orthogonal and $A$ has even degree $n d$, its determinant $\operatorname{det}\left({ }^{-}\right) \in L^{*} / L^{* 2}$ and discriminant $\operatorname{disc}\left({ }^{-}\right)=(-1)^{n d / 2} \operatorname{det}\left(^{-}\right)$are defined. If $D=L$, then $\operatorname{det}\left({ }^{-}\right)=\operatorname{det} h_{0}$, and if $D$ is a quaternion algebra, then $\operatorname{disc}\left(^{-}\right)=\operatorname{nrd}_{A / L} h_{0}$. See [1], Prop. 7.3(2),(3), p. 81.

It is useful to define as well the determinant of an involution of the second kind on a central simple algebra $A$ of even degree. In this case, with the assumption $\epsilon_{0}=1$, we define $\operatorname{det}\left({ }^{-}\right)=\operatorname{nrd}_{A / K} h_{0} \in L_{0}^{*} / \mathrm{N}_{L / L_{0}} L^{*}$. It is not difficult to check that it is well-defined, since $A$ has even degree.

We note that it follows from [14], 2.5, that a simple module $V$ over $A$ supports a nonsingular Hermitian form respectively skew Hermitian form $h: V \times V \rightarrow A$ if the simple involution algebra $\left(A,{ }^{-}\right)$is orthogonal respectively symplectic - and if $A$ is has index $>1$ or if $\left(A,{ }^{-}\right)$is unitary, it supports both. In the exceptional case when $A$ has index 1 and the involution is of the first kind, it supports only an Hermitian form if the involution is orthogonal, and only a skew Hermitian form if the involution is symplectic.

Every orthogonally indecomposable nonsingular Hermitian or skew Hermitian space $(V, h)$ over a simple hyperbolic involution algebra is hyperbolic (of length 2 ), and is uniquely determined up to equivalence.

Now let $K$ be a real closed field, and denote by $\mathbf{H}$ the unique quaternion division algebra $(-1,-1)_{K}$ over $K$. It is known that all simple components of $K G$ are stable under the canonical involution (which is the identity on $K$ ), and that for each of the three possibilities $A \cong \mathrm{M}(n, D), D=K, K(\sqrt{-1}), \mathbf{H}$, the induced involution is conjugate transpose; it is orthogonal, unitary or symplectic respectively. See for example [9] or [21], 8.13.3, p. 323.

1.3. Reformulation of the equivalence of equivariant representations. The canonical trace $\operatorname{Tr}_{K G / K}=\operatorname{Tr}: K G \rightarrow K$ is the $K$-linear functional 


$$
\operatorname{Tr}\left(\sum_{s \in G} \alpha_{s} s\right)=\alpha_{1}
$$

It is clear that this is the same thing as $\frac{1}{g} \operatorname{Tr}_{\text {reg }}$, where $\operatorname{Tr}_{\text {reg }}$ is the "algebra trace" arising from the regular representation.

If $A_{1}$ is a separable $K$-algebra, recall that $\operatorname{trd}=\operatorname{trd}_{A_{1} / K}: A_{1} \rightarrow K$ denotes the reduced trace.

Theorem 1.5. If $A \cong \mathrm{M}(n, D)$ is an arbitrary simple component of $K G$, then for all $a \in A$

$$
\operatorname{Tr}_{K G / K} a=\frac{n d}{g} \operatorname{trd}_{A / K} a .
$$

Proof. The regular trace of $A$ is the restriction of the regular trace of $K G$, and is also $n d \operatorname{trd}_{A / K}$.

Let $h: V \times V \rightarrow K$ be a (nonsingular, as always) $\epsilon$-Hermitian form with $\epsilon= \pm 1-$ if the involution on $K$ is the identity, we interpret this to mean that $h$ is symmetric or skew symmetric - and $\rho: G \rightarrow \mathbf{I}(V, h)$ an equivariant representation, $\mathbf{I}=\mathbf{O}, \mathbf{S p}$, or $\mathbf{U}$. The equivalence class of $\rho$ is determined by the equivalence class of the nonsingular $\epsilon$-Hermitian form

$$
\hat{h}: V \times V \rightarrow K G, \quad \hat{h}(u, v)=\sum_{s} h\left(\rho\left(s^{-1}\right) u, v\right) s
$$

over the group algebra $K G$ with the canonical involution (cf. [8], $\S 7$ ). Note that

$$
\operatorname{Tr}_{K G / K} \circ \hat{h}=h,
$$

and that $\hat{h}$ is the unique $\epsilon$-Hermitian form on $V$ satisfying (1.3).

Let $\left(K G,,^{-}\right)=\bigoplus_{i=1}^{r}\left(A_{i},{ }^{-}\right)$be the decomposition of $\left(K G,^{-}\right)$into simple involution components. The associated orthogonal decomposition

$$
V=A_{1} V \perp A_{2} V \perp \cdots \perp A_{r} V
$$

with respect to $h$ is also an orthogonal decomposition with respect to the form $\hat{h}$. The restriction $\hat{h}_{i}$ of $\hat{h}$ to the isotypic component $A_{i} V$ takes its values in $A_{i}$ and so can be considered as an $\epsilon$-Hermitian form $\hat{h}_{i}: A_{i} V \times A_{i} V \rightarrow A_{i}$. Since any isomorphism of $K G$-modules is an isomorphism on corresponding isotypic components, the equivalence class of the equivariant representation $\rho$ is therefore determined by the equivalence classes of the $\hat{h}_{i}$. If $A_{i}$ is a simple hyperbolic involution algebra, the equivalence class of $\hat{h}_{i}$ is completely determined by the length of the $A_{i}$-module $A_{i} V$ (see [14], $\S 2$, especially 2.3), and hence by the equivalence class of $\rho$ as a linear representation.

This means that $\rho$ is determined up to equivalence by its linear equivalence class and its isotypic subrepresentations $G \rightarrow \mathbf{I}\left(A_{i} V\right)$, where $A_{i}$ runs over the simple algebras fixed by the involution of $K G$. Suppose that these simple algebras are $A_{1}, \ldots, A_{k}$.

We can now apply the Hermitian Morita theory (8], $\S 8$, or 1.4 of this paper) to $\hat{h}_{1}, \ldots, \hat{h}_{k}$. This yields forms $\breve{h}_{1}, \ldots, \breve{h}_{k}$, where $\breve{h}_{i}$ is a symmetric, skew symmetric, Hermitian or skew Hermitian form over the division algebra which is Brauer equivalent to $A_{i}$. Once again the equivalence classes of the $\breve{h}_{i}$ characterize the equivalence class of $\rho$. Moreover, if $\rho$ is orthogonal respectively symplectic and $A_{i}$ has index 1 
with ${ }^{-}$symplectic respectively orthogonal, then $\breve{h}_{i}$ is a skew symmetric form and so is completely determined by $\operatorname{dim}_{K} A_{i} V$. Thus we can ignore such $A_{i}$ as well.

Therefore let $A_{1}, \ldots, A_{l}$ be the simple summands of $K G$ such that $\bar{A}_{i}=A_{i}$ and such that if $\rho$ is orthogonal respectively symplectic and $A_{i}$ has index 1 , then the involution ${ }^{-}$on $A_{i}$ is orthogonal respectively symplectic. Then the equivalence class of $\rho$ is determined by its equivalence class as a linear representation and by the equivalence classes of $\breve{h}_{1}, \ldots, \breve{h}_{l}$.

Another simple consequence of this procedure is that if $\rho: G \rightarrow \mathbf{G L}(V)$ is a given linear representation, there is always a $G$-invariant form $h$ of a prescribed type on $V$ if and only if the following holds for each simple component $A$ of $K G$ :

1. If $A$ is stable under the involution of $K G$ and has index 1 , then $\operatorname{len}_{A} A V$ is even if either

(i) $\left(A,{ }^{-}\right)$is symplectic and $h$ is to be symmetric, or

(ii) $\left(A,{ }^{-}\right)$is orthogonal and $h$ is to be skew symmetric.

2. $\operatorname{dim}_{K} A V=\operatorname{dim}_{K} \bar{A} V$.

Condition 1 arises from the fact that if $D$ is a division algebra with an involution, there is a nonsingular form $\breve{h}$ of any type (symmetric, skew symmetric,...) and any given rank $m \geq 1$ over $D$ unless $D$ is a field with trivial involution, $\breve{h}$ is skew symmetric, and $m$ is odd. Condition 2 results from the fact that any nonsingular $\epsilon$-Hermitian form over a hyperbolic algebra is hyperbolic ( $\S 2,[14)$.

1.4. Explicit Hermitian Morita theory and transfer theory. Suppose that $D$ is a division algebra over $K$, with an involution * which is compatible with the involution on $K$. Let $A=\mathrm{M}(n, D)$, and denote the conjugate transpose of $a \in A$ by $a^{*}$. Let $h_{0} \in \mathbf{G L}(n, D)$ be $\epsilon_{0}$-Hermitian, $h_{0}^{*}=\epsilon_{0} h_{0}$ with $\epsilon_{0}= \pm 1$. Suppose that $A$ is given the involution $\bar{a}=h_{0}^{-1} a^{*} h_{0}$.

Denote by $D^{k \times l}$ the $k \times l$ matrices over $D$, and let * again stand for conjugate transpose $D^{k \times l} \rightarrow D^{l \times k}$. $D^{n \times k}$ is a semisimple left module over $A$ of length $k$, and every (finitely generated) left module over $A$ is of this form, up to isomorphism.

Lemma 1.6. (a) The map

$$
D^{m \times k} \rightarrow \operatorname{Hom}_{A}\left(D^{n \times m}, D^{n \times k}\right)
$$

given by right multiplication is an isomorphism of $\mathrm{M}(k, D)$-modules.

(b) Multiplication

$$
D^{m \times n} \otimes_{A} D^{n \times k} \rightarrow D^{m \times k}
$$

is an isomorphism of $(\mathrm{M}(m, D), \mathrm{M}(k, D))$-bimodules.

Proof. (a) follows at once by looking at the homomorphisms $D^{n \times m} \rightarrow D^{n \times k}$ as matrices with respect to the direct sum decompositions of $D^{n \times m}$ and $D^{n \times k}$ into their "columns".

(b) Every element of $D^{1 \times n} \otimes_{A} D^{n \times 1}$ is of the form $e_{11} \otimes e_{11}^{\prime} d$, where $d \in D$ and $e_{11}$ and $e_{11}^{\prime}$ are matrix units. It follows that $D^{1 \times n} \otimes_{A} D^{n \times 1}$ is a 1-dimensional vector space over $D$, and then (b) follows easily.

Now suppose that $V=D^{n \times k}$. By the foregoing lemma, $V^{*}=\operatorname{Hom}_{A}(V, A)=$ $D^{k \times n}$. Let $f: V \times V \rightarrow\left(A,^{-}\right)$be a sesquilinear form, and consider $f_{r}: V \rightarrow$ $V^{*}, \quad f_{r}(v)=f_{r}(\cdot, v)$. If we compose this map with the map $V^{*} \rightarrow V$ given by $x \mapsto h_{0}^{-1} x^{*}$, the result is an $A$-linear homomorphism $V \rightarrow V$, and so is given by 
right multiplication by a matrix $b \in D^{k \times k}$. It follows that $f(\cdot, v)=\epsilon_{0} b^{*} v^{*} h_{0}$, and so if we set $\epsilon_{0} b^{*}=\underline{f} \in \mathrm{M}(k, D)$, we get the formula

$$
f(u, v)=u \underline{f} v^{*} h_{0} .
$$

Thus $f$ is the generalization of the notion of "discriminant matrix" (sometimes also called the "Gram matrix") of a sesquilinear form over a division ring. It is of fundamental importance in this paper.

We note that $f$ is nonsingular if and only $f$ is an invertible matrix, and $f$ is $\epsilon$-Hermitian if and only if $f^{*}=\epsilon_{0} \epsilon f$.

We give next a matrix version of Hermitian Morita theory for $A=\mathrm{M}(n, D)$. We refer to Chapter 4 of 20] for the "ordinary" Morita theory (between categories of modules) and to $\S 8$ of 8 ] for the Hermitian Morita theory.

Let $B=\mathrm{M}(m, D)$. We have perfect pairings

$$
V \times V^{*} \rightarrow A, \quad V^{*} \times V \rightarrow B,
$$

given respectively by

$$
\langle v, x\rangle=v x, \quad[x, v]=x v,
$$

satisfying the "associative laws"

$$
\langle v, x\rangle w=v[x, w], \quad x\langle v, y\rangle=[x, v] y .
$$

These data constitute a Morita context which provides a Morita equivalence between the categories ${ }_{A} \mathcal{M}$ of left $A$-modules and ${ }_{B} \mathcal{M}$ of left $B$-modules. The equivalence functors are $U \rightsquigarrow V^{*} \otimes_{A} U$ of ${ }_{A} \mathcal{M}$ into ${ }_{B} \mathcal{M}$ and $X \rightsquigarrow V \otimes_{B} X$ in the opposite direction.

If we restrict the Morita equivalence to the "skeletal" subcategory of ${ }_{A} \mathcal{M}$ of modules of the form $D^{n \times k}$, it follows from Lemma 1.6 (b), that the resulting functor is isomorphic to $D^{n \times k} \rightsquigarrow D^{m \times k}$, with the morphism $D^{n \times k} \rightarrow D^{n \times l}$ in ${ }_{A} \mathcal{M}$ given by right multiplication by a matrix in $D^{k \times l}$, being transformed into the morphism $D^{m \times k} \rightarrow D^{m \times l}$ in ${ }_{B} \mathcal{M}$ given by right multiplication by the same matrix.

Suppose that $h_{1}$ is an $\epsilon_{1}$-Hermitian form over $A$ on a simple $A$-module, where $\epsilon_{1}= \pm 1$ - for example, one can always find such an $h_{1}$ if $\epsilon_{1}=1$ unless $D=L$ and the involution ${ }^{-}$on $A$ is symplectic, i.e. $h_{0}$ is skew symmetric, in which case $h_{1}$ exists for $\epsilon_{1}=-1$ (cf. \$1.2 - this also follows easily by use of the discriminant matrix). Then an $\epsilon$-Hermitian form $\hat{h}$ over $A$ is Hermitian Morita equivalent to an $\epsilon_{1} \epsilon$-Hermitian form $\breve{h}$ over $D$, where $D$ has the adjoint involution of $h_{1}$. See [8], $\S 8$. The Hermitian Morita theory tells us that $\hat{h} \cong \hat{g}$ if and only if $\breve{h} \cong \breve{g}$.

Hermitian Morita equivalence in the case at hand is very easily described in terms of the discriminant matrix; we give it here only in the case of interest to us, when $h_{1}$ is defined on the simple module $V_{1}=D^{n \times 1}$.

Lemma 1.7. A sesquilinear form $f$ over $\left(A,{ }^{-}\right)$on $D^{n \times k}$ with discriminant matrix $\underline{f} \in \mathrm{M}(k, D)$ corresponds, via the Hermitian Morita equivalence effected by $h_{1}$, to the sesquilinear form over $\left(D,^{-}\right)$with the discriminant matrix $\epsilon_{1} f$. The involution - on $D$ is the adjoint with respect to $h_{1}$.

Proof. The discriminant matrix $\underline{h}_{1}$ of $h_{1}$ is an $\epsilon_{0} \epsilon_{1}$-Hermitian element of $\left(D,{ }^{*}\right)$, i.e. $\underline{h}_{1}^{*}=\epsilon_{0} \epsilon_{1} \underline{h}_{1}$. The adjoint involution on $D$ with respect to $h_{1}$ is $\bar{d}=\underline{h}_{1} d^{*} \underline{h}_{1}^{-1}$.

Let $\bar{V}_{1}$ be the $(A, D)$-bimodule $V_{1}$ twisted into a $(D, A)$-bimodule by the involution ${ }^{-}$on both $A$ and $D$. The $\epsilon_{1}$-Hermitian form $H: \bar{V} \times \bar{V} \rightarrow\left(D,^{-}\right)$which effects 
the Morita equivalence between the categories of sesquilinear forms over $\left(A,{ }^{-}\right)$and $\left(D,{ }^{-}\right)$respectively is defined by

$$
h_{1}\left(v_{1}, v_{2}\right) v_{3}=v_{1} H\left(\bar{v}_{2}, \bar{v}_{3}\right) \quad \text { for all } v_{1}, v_{2}, v_{3} \in V
$$

(cf. (8.3), [8]), where $\bar{v}_{i}$ stands for the vector $v_{i} \in V$ considered as a vector of $\bar{V}_{1}$. Since $h_{1}\left(v_{1}, v_{2}\right)=v_{1} \underline{h}_{1} v_{2}^{*} h_{0}$, this implies that

$$
H\left(\bar{v}_{2}, \bar{v}_{3}\right)=\underline{h}_{1} v_{2}^{*} h_{0} v_{3} .
$$

This is the analogue of (1.4) for a form on a right module instead of a left module. Thus the discriminant matrix of $H$ is $h_{0}$.

We identify the twisted bimodule $\bar{V}_{1}$ with the $A$-dual $V_{1}^{*}=D^{1 \times n}$ via the $(A, D)$ bimodule isomorphism $h_{1 r}$ which takes $\bar{v}$ to $h_{1}(\cdot, v)=\underline{h}_{1} v^{*} h_{0}$.

Now let $(U, f)$ be a sesquilinear form over $\left(A,{ }^{-}\right)$, where $U=D^{n \times k}$, and suppose that the discriminant matrix of $f$ is $f$. We wish to find the discriminant matrix of the sesquilinear form $\left(\bar{V}_{1} \otimes_{A} U, H f\right)$ over $D$ which corresponds to $(U, f)$ under the Hermitian Morita equivalence effected by $h_{1}$ and $H$. Both of the mappings

$$
\bar{V}_{1} \otimes_{A} U \stackrel{h_{1 r} \otimes \text { idu }}{\longrightarrow} V_{1}^{*} \otimes_{A} U \stackrel{\text { mult. }}{\longrightarrow} D^{1 \times k}
$$

are isomorphisms of left $D$-spaces, and their composition takes $\bar{v} \otimes u$ to $\underline{h}_{1} v^{*} h_{0} u \in$ $D^{1 \times k}$. We identify $\bar{V} \otimes_{A} U$ and $D^{1 \times k}$ by this isomorphism.

Now by definition of the product form $H f$,

$$
H f\left(\bar{v}_{1} \otimes u_{1}, \bar{v}_{2} \otimes u_{2}\right)=H\left(\bar{v}_{1} f\left(u_{1}, u_{2}\right), \bar{v}_{2}\right)=H\left(\overline{\overline{f\left(u_{1}, u_{2}\right)}} v_{1}, \bar{v}_{2}\right) .
$$

A straightforward computation shows that

$$
H f\left(\bar{v}_{1} \otimes u_{1}, \bar{v}_{2} \otimes u_{2}\right)=\epsilon_{1}\left(\bar{v}_{1} \otimes u_{1}\right) \underline{f}\left(\bar{v}_{2} \otimes u_{2}\right)^{*} h_{1}^{-1},
$$

which implies that the discriminant matrix of $H f$ is $\epsilon_{1} \underline{f}$.

This explicit version of the Hermitian Morita theory underlies our computation of the equivalence classes of equivariant representations in the rest of the paper, and we now describe the version of it which will be used.

If the involution on $A=\mathrm{M}(n, D)$ is of the first kind and $D$ is a quaternion algebra, we choose it to be conjugation * .

Now choose $h_{1}: V_{1} \times V_{1} \rightarrow A$ to be the form $h_{1}\left(u_{1}, v_{1}\right)=u_{1} v_{1}^{*} h_{0}$. Then $h_{1}$ is $\epsilon_{0}$-Hermitian with respect to ${ }^{-}$and its adjoint involution on $D$ is the involution - chosen above; in particular it is conjugation if it is of the first kind and $D$ is a quaternion algebra.

We now apply Lemma [1.7 to get:

Lemma 1.8. With the foregoing notation, the $\epsilon$-Hermitian form

$$
\hat{h}: D^{n \times m} \times D^{n \times m} \rightarrow A
$$

with discriminant matrix $\underline{\hat{h}} \in D^{m \times m}$ (which is $\epsilon_{0} \epsilon$-Hermitian),

$$
\hat{h}(u, v)=u \underline{\hat{h}} v^{*} h_{0},
$$

corresponds via the Hermitian Morita theory arising from $h_{1}$ to the $\epsilon_{0} \epsilon$-Hermitian form

$$
\breve{h}: D^{1 \times m} \times D^{1 \times m} \rightarrow D
$$


which has discriminant matrix $\epsilon_{0} \underline{\hat{h}}$,

$$
\breve{h}(u, v)=u \epsilon_{0} \underline{\hat{h}} v^{*} .
$$

And if $D$ is a quaternion algebra and the involution on $A$ is of the first kind, the involution on $D$ is conjugation.

Remark 1.9. In the exceptional case when $\left(A,{ }^{-}\right)$is symplectic of index 1 , the correspondence between forms over $A$ and over $L=\operatorname{cen} A$ under which the form over $A$ with matrix $f$ corresponds to the form over $L$ with matrix $f$ is a category equivalence, since for example the functor $(W, g) \rightsquigarrow(W,-g)$ is certainly an automorphism of the category of bilinear forms over $L$. But this correspondence does not arise from a Morita equivalence.

We now use the discriminant matrix to compute the transfer $\operatorname{trd}_{A / L} \hat{h}$ of the $\epsilon$-Hermitian form $\hat{h}$ over $A$; the transfer is an $\epsilon$-Hermitian form over $L$ (symmetric or skew symmetric if ${ }^{-}$is the identity on $\left.K\right)$.

We note that if $a=\left(a_{i j}\right) \in A$, then

$$
\operatorname{trd}_{A / K} a=\sum_{i} \operatorname{trd}_{D / K} a_{i i}=\operatorname{trd}_{D / K} \sum_{i} a_{i i} .
$$

Define $\operatorname{trd}_{A / D} a=\sum_{i} a_{i i}$. This depends on the identification $A=\mathrm{M}(n, D)$, but is nevertheless useful. The above formula becomes

$$
\operatorname{trd}_{A / K}=\operatorname{trd}_{D / K} \circ \operatorname{trd}_{A / D}
$$

A straightforward matrix computation proves:

Lemma 1.10. Suppose that $D=L$. The discriminant matrix of $\operatorname{trd}_{A / L} \hat{h}$ (in a suitable basis) is

$$
\underline{\hat{h}} \otimes h_{0}^{t}
$$

and its determinant is

$$
(\operatorname{det} \underline{\hat{h}})^{n}\left(\operatorname{det} h_{0}\right)^{m} .
$$

Now consider the case where $A$ has index $>1$, i.e. $D$ is not commutative. We can suppose that $h_{0}$ and $\underline{\hat{h}}$ are diagonal matrices, say $h_{0}=\left\langle\eta_{01}, \ldots, \eta_{0 n}\right\rangle$ and $\underline{\hat{h}}=\left\langle\eta_{1}, \ldots, \eta_{m}\right\rangle$. Note that for all $i$ and $j, \eta_{0 i}^{*}=\epsilon_{0} \eta_{0 i}$ and $\eta_{j}^{*}=\epsilon_{0} \epsilon \eta_{j}$. The one dimensional form

$$
H_{i j}: D \times D \rightarrow D, \quad H_{i j}(u, v)=u \eta_{j} v^{*} \eta_{0 i}, \quad 1 \leq i \leq n, 1 \leq j \leq m,
$$

is an $\epsilon$-Hermitian form with respect to the involution

$$
d \mapsto \eta_{0 i}^{-1} d^{*} \eta_{0 i}
$$

of $D$. Another matrix computation proves:

Lemma 1.11. (a) The transfer $\operatorname{trd}_{A / L} \hat{h}: D^{n \times m} \times D^{n \times m} \rightarrow L$ is an $\epsilon$-Hermitian form and is the orthogonal direct sum of the transfers from $D$ to $L$ of the $n m$ forms $H_{i j}$ in (1.9), where $H_{i j}$ is a one dimensional $\epsilon$-Hermitian form with respect to the involution (1.10).

(b) Suppose that $h_{0}=\eta_{0} \mathrm{I}_{n}$ (for example, $h_{0}=\mathrm{I}_{n}$ ). Then the transfer

$$
H=\operatorname{trd}_{A / D} \hat{h}: D^{n \times m} \times D^{n \times m} \rightarrow D
$$


is a nonsingular $\epsilon$-Hermitian form with respect to the involution

$$
\bar{d}=\eta_{0}^{-1} d^{*} \eta_{0}
$$

and its transfer to $L$ is the transfer of $\hat{h}$ to $L$,

$$
\operatorname{trd}_{D / L} H=\operatorname{trd}_{A / L} \hat{h} .
$$

Its discriminant matrix $\underline{H} \in D^{n m \times n m}$, defined by $H(u, v)=u \underline{H} v^{*} \eta_{0}, u, v \in$ $D^{1 \times n m}$, is given by

$$
\underline{H}=\underline{\hat{h}} \otimes h_{0} .
$$

If $K$ is a non-Archimedean local field and $D$ is the unique quaternion algebra over $K$, with conjugation * as its involution, it is always possible to achieve $h_{0}=\eta \mathrm{I}_{n}$ except in the case $n$ even and $h_{0}^{*}=-h_{0}$. See Table 1 in $\S 1$.

The next lemma is useful in calculating the transfers of the $H_{i j}$.

Let $D$ be a central quaternion division algebra over $K$ with an orthogonal involution ${ }^{-}$. There is a pure quaternion $j$ such that $\bar{d}=j^{-1} d^{*} j$ for all $d \in D$, where * denotes conjugation in $D$ (cf. 11.2 ).

Thus if $h: D \times D \rightarrow\left(D,^{-}\right)$is a rank 1 Hermitian form, there is another pure quaternion $\eta$ such that

$$
h\left(d_{1}, d_{2}\right)=d_{1} \eta d_{2}^{*} j=d_{1} \eta j \bar{d}_{2} .
$$

With this notation, we have:

Lemma 1.12. The quaternary quadratic form $\operatorname{trd}_{D / K} h$ over $K$ has determinant 1 , and is isotropic (hence hyperbolic) if and only if $\left(\eta^{2}, j^{2}\right)_{K} \cong D$.

Proof. Conjugation by $j$ of the space $D_{0}$ of pure quaternions is an involutory linear transformation, and it follows, by consideration of the eigenspaces, that we can find another pure quaternion $i \neq 0$ satisfying $\eta=\theta j+\delta i$ with $\theta \in K, i j=-j i$, and $\delta=0$ or 1 .

Let $i^{2}=\alpha, j^{2}=\beta$, so $D=(\alpha, \beta)_{K}$. And

$$
\eta^{2}=\theta^{2} \beta+\delta^{2} \alpha
$$

The discriminant matrix of $\operatorname{trd}_{D / L} h$ in the basis $1, k=i j, i, j$ of $D$ is

$$
2 \beta\left(\begin{array}{cccc}
\theta & -\alpha \delta & 0 & 0 \\
-\alpha \delta & -\theta \alpha \beta & 0 & 0 \\
0 & 0 & \theta \alpha & -\alpha \delta \\
0 & 0 & -\alpha \delta & -\theta \beta
\end{array}\right) .
$$

Its determinant is $4 \beta^{2}\left(-\theta^{2} \alpha \beta-\alpha^{2} \delta^{2}\right)^{2}=1$ in $K^{*} / K^{* 2}$.

If $\theta=0$, a glance at the above matrix shows that $\operatorname{trd}_{D / K} h$ is isotropic; furthermore $\eta=i$, so $\left(\eta^{2}, j^{2}\right)_{K}=D$.

Suppose $\theta \neq 0$. The forms $b_{1}$ and $b_{2}$ with matrices

$$
\left(\begin{array}{cc}
\theta & -\alpha \delta \\
-\alpha \delta & -\theta \alpha \beta
\end{array}\right) \text { and }\left(\begin{array}{cc}
\theta \alpha & -\alpha \delta \\
-\alpha \delta & -\theta \beta
\end{array}\right)
$$

have the same determinant, and so their sum $b_{1} \perp b_{2}$ is isotropic if and only if $b_{2} \cong-b_{1}$. This is equivalent to the form $\left\langle\theta,-\theta^{3} \alpha \beta-\theta \alpha^{2} \delta^{2}\right\rangle$ representing $\theta \beta$, hence to $\left\langle 1,-\theta^{2} \alpha \beta-\alpha^{2} \delta^{2}\right\rangle$ representing $\beta$. Since binary forms are classified by their determinant and Hasse symbol, this in turn is equivalent to

$$
\left(\beta,-\theta^{2} \alpha \beta^{2}-\alpha^{2} \delta^{2} \beta\right)_{K}=\left(1,-\theta^{2} \alpha \beta-\alpha^{2} \delta^{2}\right)_{K}=1
$$


i.e. to $\left(\beta, \theta^{2} \alpha \beta+\alpha^{2} \delta^{2}\right)_{K}=1$. Thus the condition is

$$
1=(\beta, \alpha)_{K}\left(\beta, \theta^{2} \beta+\alpha \delta^{2}\right)_{K}=(\beta, \alpha)_{K}\left(j^{2}, \eta^{2}\right)_{K},
$$

i.e. $D=(\alpha, \beta)_{K} \cong\left(\eta^{2}, j^{2}\right)_{K}$.

\section{Symplectic Representations}

Because of the fact that two nonsingular skew symmetric forms are equivalent if and only if they have the same rank, symplectic representations are considerably easier to deal with than are orthogonal or unitary representations, and so we treat them separately here.

We now prove the statements on symplectic representations given earlier.

We identify $A=\mathrm{M}(n, D)$ and $V=D^{n \times m}$. The involution on $A$ is of the form $\bar{a}=h_{0}^{-1} a^{*} h_{0}-$ see $\$ 1.2$

If $\underline{\hat{h}}$ is an $m \times m$ matrix, the form $h(u, v)=\operatorname{Tr}_{A / K}\left(u \underline{\hat{h}} v^{*} h_{0}\right)$ on $D^{n \times m}$ will be skew symmetric if and only if $\hat{h}(u, v)=u \underline{\hat{h}} v^{*} h_{0}$ is skew Hermitian.

Suppose that $\left(A,{ }^{-}\right)$is orthogonal and of index 1 . Then $\underline{\hat{h}}$ must be skew symmetric (in order for $h$ to be skew symmetric). This verifies II (in the orthogonal case) for any field $K$ if $\left(A,^{-}\right)$has index 1 . This is always the case if $K$ is finite or real closed - see $\$ 1.2$ - which proves I for these fields (by Table 1).

Suppose next that $\left(A,{ }^{-}\right)$is orthogonal but has index $>1$. Recall that we assume that the involution ${ }^{*}$ on $D$ is symplectic when $D \neq L$. Since $h_{0}$ is then skew Hermitian, $\underline{\hat{h}}$ must be an Hermitian matrix, so the equivalence classes of symplectic representations on $V$ are in bijective correspondence with the equivalence classes of Hermitian forms of rank $m$ over $D$ by Hermitian Morita theory. There is only 1 when $K$ is local, by Table 1 .

Now suppose that $K$ is global. We must count the number of Hermitian forms $\breve{h}$ of rank $m$ over $D$, a quaternion division algebra with the canonical involution. If $D$ splits at the prime $\mathfrak{p}$ of its center $L, \breve{h}_{\mathfrak{p}}$ is an Hermitian form over $\mathrm{M}\left(2, L_{\mathfrak{p}}\right)$ with a symplectic involution. Thus we can assume that $\breve{h}_{\mathfrak{p}}(u, v)=u \underline{\hat{h}} v^{t} h_{1}$, where $h_{1}$ is a skew symmetric matrix of degree 2 . Therefore, as a $2 m \times 2 m$ matrix over $L_{\mathfrak{p}}, \underline{\hat{h}}$ is skew symmetric and so is uniquely determined up to equivalence.

We note that $D$ does split at every real prime $\mathfrak{p}$ of the center, since $\left(A,^{-}\right)$is orthogonal - cf. 1.2

If $D$ is not split at the finite prime $\mathfrak{p}, \breve{h}_{\mathfrak{p}}$ is an Hermitian form over the local division algebra $D_{\mathfrak{p}}$ and so is again uniquely determined up to equivalence by its rank (cf. Table 1). By the Hasse Principle for Hermitian forms over $D$ (cf. Table $1)$, this finishes the proof for $\left(A,^{-}\right)$orthogonal.

Suppose now that $\left(A,{ }^{-}\right)$is symplectic. This means that $h_{0}$ is skew symmetric if $A$ has index 1 and is Hermitian otherwise. Then $h(u, v)=\operatorname{trd}_{A / K}\left(u \underline{\hat{h}} v^{*} h_{0}\right)$ is an invariant skew symmetric form on $D^{n \times m}$ if and only if $\underline{\hat{h}}$ is nonsingular and symmetric in the first case and skew Hermitian in the second. The cases of $K$ finite, or $K$ global, or $K$ local and $A$ of index 1 follow easily. If $K$ is real closed, $A$ is necessarily of index $>1$ (1.2); by Table 1 , skew Hermitian forms over $D$ are classified by their rank and so there is only 1 equivalence class of symplectic representations in this case. Finally, the case of $K$ local and $\left(A,^{-}\right)$of index $>1$ follows from [21], Theorem 10.3.6. 
The case $\left(A^{-}\right)$unitary is proved in a similar manner. Note that (noncommutative) division algebras over local or real closed fields do not admit involutions of the second kind - in the real closed case, $A$ admits a unitary involution if and only if $A$ is a full matrix algebra over the algebraic closure of $K$, again by 1.2

Now III. If $G$ is a symmetric group, it is well known that all of the simple components of $K G$ are split and stable under the canonical involution, and that the induced involution in each case is orthogonal (cf. \$6). This proves III in this case (by II). Now consider 2, 3 and 4. The assumption of odd representation type rules out both a symplectic involution and an orthogonal involution on an algebra of Schur index $>1$. So III is clear if $K$ is finite. Otherwise conditions 3 and 4 both rule out a unitary involution which is not hyperbolic. Thus each simple component $A$ of $K G$, stable under the canonical involution and for which $A V \neq 0$, is orthogonal of index 1 .

Finally, consider the statement on conjugacy of subgroups of $\mathbf{S p}(V, h)$. Since $G$ and $G^{\prime}$ are conjugate in $\mathbf{G L}(V)$, there is a $\phi \in \mathbf{G} \mathbf{L}(V)$ such that $s \mapsto \phi^{-1} s \phi$ is an isomorphism $G \rightarrow G^{\prime}$. This map and the identity are symplectic representations which are linearly equivalent, so by III we can find $\psi \in \mathbf{S p}(V, h)$ such that $s \rightarrow$ $\psi^{-1} s \psi$ is an isomorphism $G \rightarrow G^{\prime}$.

\section{UNITARY AND ORTHOGONAL REPRESENTATIONS OVER FINITE FIELDS}

If $K$ is a finite field, the simple algebra $A$ is a matrix algebra $\mathrm{M}(n, L)$ over the field $L$.

Theorem 3.1. Let $K$ be a finite field, $A$ a simple $K$-algebra and $V$ an $A$-module of length $\operatorname{len}_{A} V=m$.

Let $h: V \times V \rightarrow K$ be a symmetric, Hermitian or skew Hermitian form.

\section{I, II.}

Unitary representations. There is exactly 1 equivalence class of $A$-isotypic unitary representations $G \rightarrow \mathbf{U}(V, h)$, in both the Hermitian and skew Hermitian cases.

Orthogonal representations. If $h$ is symmetric, then the number of equivalence classes of A-isotypic orthogonal representations $G \rightarrow \mathbf{O}(V, h)$ is

(a) 1 if $\left(A,{ }^{-}\right)$is symplectic, $m$ is even and $h$ is hyperbolic,

(b) 1 if $\left(A,,^{-}\right)$is orthogonal and $n$ is odd,

(c) 2 if $\left(A,{ }^{-}\right)$is orthogonal, $n$ is even and $\operatorname{det} h=\mathrm{N}_{L / K}\left(\operatorname{det}\left(^{-}\right)\right)^{m}$,

(d) 1 if $\left(A,{ }^{-}\right)$is unitary and $\operatorname{det} h=\left(-\operatorname{disc}\left(L / L_{0}\right)\right)^{m n\left|L_{0}^{*}\right| /\left|K^{*}\right|}$,

and is 0 otherwise.

\section{III.}

Unitary representations. Two unitary representations of $G$ over $K$ are equivalent if and only if they are linearly equivalent.

Orthogonal representations. Two orthogonal representations of $G$ over $K$ are equivalent if and only if they are linearly equivalent and their forms are isotypically equivalent, unless $K G$ has a simple orthogonal component $\left(A,{ }^{-}\right)$of even degree for which $A V \neq 0$.

If $\rho: G \rightarrow \mathbf{O}(V, h)$ is an orthogonal representation and $r$ is the number of orthogonal components $\left(A^{-}\right)$of even degree for which $A V \neq 0$, the number of 
equivalence classes of orthogonal representations $G \rightarrow \mathbf{O}(V, h)$ which are linearly equivalent to $\rho$ is $2^{r}$.

In particular, two orthogonal representations of $G$ of odd type are equivalent if and only if they are equivalent as linear representations and have isotypically equivalent forms.

Corollary 3.2. If $K$ is a finite field, two subgroups of $\mathbf{U}(V, h)$ which have order relatively prime to char $K$ are conjugate in $\mathbf{U}(V, h)$ if and only if they are conjugate in $\mathbf{G L}(V)$.

Proof of the theorem. We view the succession of equivalences $\rho \rightsquigarrow \hat{h} \rightsquigarrow \breve{h}$ (cf. $₫ 1.3$ ) in the reverse direction. Thus we consider all possibilities for $\breve{h}$ (up to equivalence) and $\left(A,{ }^{-}\right)$, and for each such pair $\breve{h},\left(A,{ }^{-}\right)$we determine the form $h$ by first determining $\hat{h}$ by Hermitian Morita theory using Lemma 1.8 and then $h=\frac{n}{g} \operatorname{trd}_{A / K} \hat{h}$ via the transfer theory using Lemma 1.10. The equivalence classes of $h$ 's so obtained are those whose forms which admit an equivariant representation linearly equivalent to $\rho$, and the number of equivalence classes of $\breve{h}$ 's which lead to the equivalence class of $h$ is the number of nonequivalent orthogonal representations $G \rightarrow \mathbf{O}(V, h)$ or unitary representations $G \rightarrow \mathbf{U}(V, h)$.

We use the notation of Lemma 1.8 . The form $\breve{h}$ is a symmetric, skew symmetric, Hermitian or skew Hermitian form over $L$ on $L^{m}$ with matrix $\epsilon_{0} \underline{\hat{h}}$.

Suppose first that $\breve{h}$ is skew symmetric - in particular, $m$ is even and the involution on $K$ is the identity. If the involution on $A$ is orthogonal, the form $\hat{h}$ is skew Hermitian and the form $h$ must be skew symmetric. This case is already handled in 92

If $\breve{h}$ is skew symmetric and the involution on $A$ is symplectic, $\hat{h}$ is an Hermitian form over $A$ on $L^{n \times m}$, and its transfer $h^{\prime}=\operatorname{trd}_{A / L} \hat{h}$ is a symmetric form over $L$ on $L^{n \times m}$ with matrix $\underline{\hat{h}} \otimes h_{0}^{t}$ (cf. \$1.4 $)$ which, since $h_{0}$ and $\underline{\hat{h}}$ are both skew symmetric, is hyperbolic. Thus $\operatorname{Tr}_{L / K}\left(h^{\prime}\right)=\operatorname{trd}_{A / K} \hat{h}$ is also hyperbolic, and so also $h=\operatorname{Tr} \hat{h}=\frac{n}{g} \operatorname{trd}_{A / K} \hat{h}$. This is (a).

Now suppose that $\breve{h}$ is symmetric. If the involution on $A$ is symplectic, $\hat{h}$ is skew Hermitian, so $\operatorname{Tr} \hat{h}=\frac{n}{g} \operatorname{trd}_{A / K} \hat{h}$ is skew symmetric and can be disregarded.

If $\breve{h}$ is symmetric and the involution is orthogonal, $\hat{h}$ is Hermitian, and $\operatorname{trd}_{A / L} \hat{h}$ is symmetric; moreover by the determinant formula $\operatorname{det}\left(\operatorname{trd}_{A / L} \hat{h}\right)=(\operatorname{det} \underline{\hat{h}})^{n}\left(\operatorname{det} h_{0}\right)^{m}$ (cf. (1.8), the two possibilities for $\breve{h}$ (up to equivalence) give rise to two nonequivalent transfers $\operatorname{trd}_{A / L} \hat{h}$ if $n$ is odd, while if $n$ is even there is only one possibility for $\operatorname{trd}_{A / L} \hat{h}$, up to equivalence. Moreover in the latter case, $\operatorname{trd}_{A / L} \hat{h}$ has determinant $\left(\operatorname{det} h_{0}\right)^{m}=\left(\operatorname{det}\left(^{-}\right)\right)^{m}$. Since the norm $\mathrm{N}_{L / K}$ preserves nonsquares, by Lemma 1.1 the same thing is true for $\operatorname{trd}_{A / K} \hat{h}=\operatorname{Tr}_{L / K} \operatorname{trd}_{A / L} \hat{h}=\frac{g}{n} \operatorname{Tr}_{K G / K} \hat{h}=\frac{g}{n} h$ (namely two nonequivalent forms when $n$ is odd, and one when $n$ is even), so also for $h$, where $h$ has determinant $\left(\frac{n}{g}\right)^{l m n} \mathrm{~N}_{L / K}\left(\operatorname{det} h_{0}\right)^{m}=\mathrm{N}_{L / K}\left(\operatorname{det}\left({ }^{-}\right)\right)^{m}$ in $K^{*} / K^{* 2}$ when $n$ is even. This gives (b) and (c).

Now suppose that the involution on $A$ is of the second kind, so we can take $h_{0}=\mathrm{I}_{n}$ since Hermitian forms over a finite field are characterized by their dimension (cf. Table 1). Consider first the case where the involution on $K$ is the identity. If $\breve{h}$ is skew Hermitian, $h$ is skew symmetric. If $\breve{h}$ is Hermitian, we get one class of orthogonal representations for the form $h$ since there is only one equivalence class of 
Hermitian forms of a given rank. Furthermore if $L=L_{0}\left(\sqrt{\lambda_{0}}\right), h$ has discriminant

$$
\mathrm{N}_{L_{0} / K}\left(-\lambda_{0}\right)^{m n}=\left(-\lambda_{0}\right)^{\left|L_{0}^{*}\right| /\left|K^{*}\right|}
$$

by Lemma 1.1 since $\operatorname{trd}_{\mathrm{A} / \mathrm{L}} \hat{\mathrm{h}}$ has matrix $\mathrm{I}_{m} \otimes \mathrm{I}_{n}$ and the discriminant of the binary symmetric form $\operatorname{Tr}_{L / L_{0}}(x \bar{y})$ on $L$ is $-\lambda_{0}$. This is (d).

Now suppose the involution on $K$ is also not the identity. There is only one equivalence class of skew Hermitian forms on $L^{m}$, and it gives rise to a single equivalence class of unitary representations (with respect to a skew Hermitian form). Similarly we get a single equivalence class of unitary representations with respect to an Hermitian form from the unique equivalence class of Hermitian forms on $L^{m}$.

III is clear from $\mathbf{I}$ and $\mathbf{I I}$.

\section{UNITARY AND ORTHOGONAL REPRESENTATIONS OVER LOCAL FIELDS}

If $\mathcal{A}$ is an Abelian group, $\sigma_{2}(\mathcal{A})$ denotes the number of square classes $\left(\mathcal{A}: \mathcal{A}^{2}\right)$. In this section $K$ is a local (non-Archimedean) field. It is "dyadic" if 2 is not a unit, "nondyadic" otherwise, and

$$
\sigma_{2}\left(L^{*}\right)=\left(L^{*}: L^{* 2}\right)=\left\{\begin{array}{cl}
4 & \text { if } L \text { is nondyadic } \\
2^{2+\left(L: \mathbb{Q}_{2}\right)} & \text { if } L \text { is dyadic }
\end{array}\right.
$$

(21], p. 217). Let $g_{K}$ denote the unique quaternary anisotropic form over $K$, and $\mathcal{G}$ the (Abelian) Galois group $\operatorname{Gal}(L / K)$.

The integer $m^{\prime}$ in part $(\mathrm{k})$ of the next theorem is

$$
m^{\prime}= \begin{cases}2 \quad & \text { if } m=1 \text { and either } n \text { is odd and } h \sim_{W} 0 \\ & \text { or } n \text { is even and } h \sim_{W} g_{K} \\ m & \text { otherwise }\end{cases}
$$

Theorem 4.1. Let $K$ be a local field, $A \cong \mathrm{M}(n, D)$ a simple algebra component of $K G$ stable under ${ }^{-}$, and $V$ an $A$-module of length $\operatorname{len}_{A} V=m$.

Let $h: V \times V \rightarrow K$ be a symmetric or Hermitian form.

Unitary representations. The number of equivalence classes of A-isotypic unitary representations $G \rightarrow \mathbf{U}(V, h)$ is
type of
$\left(A,{ }^{-}\right)$
\# of equiv.
classes
(a) unitary
1
$n$ odd,
(b) unitary
2

\section{conditions}

and 0 otherwise.

Orthogonal representations. The number of equivalence classes of A-isotypic orthogonal representations $G \rightarrow \mathbf{O}(V, h)$ is 


\begin{tabular}{|c|c|c|c|}
\hline & type of & \# of equiv. & conditions \\
\hline (c) & unitary & 1 & $n$ odd and $\operatorname{det} h=\mathrm{N}_{L_{0} / K}\left(-\operatorname{disc}\left(L / L_{0}\right)\right)^{m}$, \\
\hline (d) & unitary & 2 & $n$ even, and \\
\hline & & & $\begin{array}{l}h \sim_{W} 0 \text { if }\left(\operatorname{det}\left({ }^{-}\right)\right)^{m}=(-1)^{m n / 2} \\
h \sim_{W} g_{K} \text { otherwise }\end{array}$ \\
\hline (e) & sympl. & 1 & $\begin{array}{l}h \sim_{W} g_{K} \text { if } m n \text { is odd, otherwise } h \sim_{W} 0 \\
\text { and, if } D=L, m \text { is even, }\end{array}$ \\
\hline$(f)$ & orthog., & $\frac{\sigma_{2}(\mathcal{G}) \sigma_{2}\left(L^{*}\right)}{\sigma_{2}\left(K^{*}\right)}$ & $\begin{array}{l}n \text { odd, } m>1, \operatorname{det} h \in \\
\qquad(\operatorname{disc}(L / K))^{m}\left(\mathrm{~N}_{L / K} L^{*}\right) K^{* 2}\end{array}$ \\
\hline (g) & $\begin{array}{l}\text { ind. }=1 \\
\text { orthog., } \\
\text { ind. }=1\end{array}$ & $\begin{array}{c}1 \leq \# \\
\leq \frac{\sigma_{2}(\mathcal{G}) \sigma_{2}\left(L^{*}\right)}{\sigma_{2}\left(K^{*}\right)}\end{array}$ & $n$ odd, $m=1, h \cong \operatorname{Tr}_{L / K}\left\langle\alpha h_{0}\right\rangle, \alpha \in L^{*}$, \\
\hline (h) & $\begin{array}{l}\text { orthog., } \\
\text { ind. }=1\end{array}$ & $\frac{\sigma_{2}\left(L^{*}\right)}{1+\delta_{1 m}}$ & $n$ even, $\operatorname{disc}\left({ }^{-}\right) \neq 1, \operatorname{det} h=\mathrm{N}_{L / K}\left(\operatorname{det}\left({ }^{-}\right)\right)^{m}$ \\
\hline (i) & $\begin{array}{l}\text { orthog., } \\
\text { ind. }=1\end{array}$ & $\frac{2 \sigma_{2}\left(L^{*}\right)}{1+\delta_{1 m}}$ & $\begin{array}{l}n \text { even, } \operatorname{disc}(-)=1, h \sim_{W} 0 \text { or } g_{K} \\
\text { if } m n / 2 \text { is even, } \\
\quad h \sim_{W} 0 \Leftrightarrow \mathrm{S}\left(h_{0}\right)^{m}=(-1,-1)_{L}^{m^{2} n / 4} \\
\text { if } m n / 2 \text { is odd, }\end{array}$ \\
\hline (j) & $\begin{array}{l}\text { orthog., } \\
\text { ind. }>1\end{array}$ & $\sigma_{2}\left(L^{*}\right)-\delta_{1 m}$ & $\begin{array}{l}\quad h \sim_{W} 0 \Leftrightarrow \mathrm{S}\left(h_{0}\right)^{m}=(-1,-1)_{L}^{\left(m^{2} n-2\right) / 4} \\
\operatorname{det}(-)=1, h \sim_{W} 0 \text { if } m n \text { is even, } \\
h \sim_{W} g_{K} \text { if } m n \text { is odd, }\end{array}$ \\
\hline (k) & $\begin{array}{l}\text { orthog., } \\
\text { ind. }>1\end{array}$ & $\frac{1}{2} \sigma_{2}\left(L^{*}\right)-\delta_{1 m^{\prime}}$ & $\operatorname{det}\left({ }^{-}\right) \neq 1, h \sim_{W} 0$ or $g_{K}$ \\
\hline
\end{tabular}

and 0 otherwise.

Remark 4.2. 1. In (f) and (g), $\sigma_{2}\left(L^{*}\right)=\sigma_{2}\left(K^{*}\right)$ if $K$ is nondyadic, $\sigma_{2}(\mathcal{G})=1$ and $\left(\mathrm{N}_{L / K} L^{*}\right) K^{* 2}=K^{*}$ if $(L: K)$ is odd.

2. In (g), $\left\langle\alpha h_{0}\right\rangle$ is the symmetric form of rank $n$ and matrix $\alpha h_{0}$. The determinant of its trace $\operatorname{Tr}_{L / K}\left\langle\alpha h_{0}\right\rangle$ is $\operatorname{disc}(L / K) \mathrm{N}_{L / K}\left(\alpha \operatorname{det} h_{0}\right)$. Its Hasse symbol can be calculated using [6], Lemma 1, 3, but the result unfortunately involves the unknown factor $\mathrm{S}_{K}\left(\left\langle\alpha\right.\right.$ disc $\left.\left.h_{0}\right\rangle\right)$, the Hasse symbol of a "scaled trace form" of the extension $L / K$. It is possible, however, to give complete results when $K$ is a nondyadic field; this is carried out in Theorem 4.9 .

We note that the discriminants of the $h$ in this case are

$$
\left(\frac{n}{g}\right)^{l}(\operatorname{disc}(L / K))\left(\mathrm{N}_{L / K} L^{*}\right) K^{* 2}
$$

and, by Remark 1, the number of equivalence classes is 1 if $l$ is odd and $K$ is nondyadic, or if $A$ is split.

3. In (i), $\mathrm{S}\left(h_{0}\right)$ is well-defined. In fact, by Lemma 1.3 (i), if $\eta \in L^{*}$ then

$$
\mathrm{S}\left(\eta h_{0}\right)=\mathrm{S}\left(h_{0}\right)(\eta,-1)_{L}^{n(n-1) / 2}\left(\eta,(-1)^{n(n-1) / 2}\right)_{L}=\mathrm{S}\left(h_{0}\right),
$$

since disc $h_{0}=1$ implies that $\operatorname{det} h_{0}=(-1)^{n(n-1) / 2}$.

4. The integer $m$ can be calculated easily from a knowledge of $h$ and $\left(A,{ }^{-}\right)$. Namely, the rank of $h$ is $m n d^{2} l$. 
Proof. The proof proceeds in the same way as that of Theorem 3.1, starting with the form

$$
\breve{h}: D^{m} \times D^{m} \rightarrow D
$$

and then "descending" first to

$$
\hat{h}: D^{n \times m} \times D^{n \times m} \rightarrow \mathrm{M}(n, D)=A
$$

via Morita theory using Lemma 1.8 then to

$$
\operatorname{trd}_{A / D} \hat{h}: D^{n m} \times D^{n m} \rightarrow D
$$

via a transfer from $A$ to $D$, then to

$$
\operatorname{trd}_{D / L} \operatorname{trd}_{A / D} \hat{h}=\operatorname{trd}_{A / L} \hat{h}: L^{d^{2} n m} \times L^{d^{2} n m} \rightarrow L
$$

(where $d^{2}=\operatorname{dim}_{L} D=1$ or 4 ) via a transfer from $D$ to $L$, and then finally to

$$
\frac{n d}{g} \operatorname{trd}_{L / K} \operatorname{trd}_{A / L} \hat{h}=\frac{n d}{g} \operatorname{trd}_{A / K} \hat{h}=\operatorname{Tr}_{K G / K} \hat{h}=h: K^{l d^{2} n m} \times K^{l d^{2} n m} \rightarrow K
$$

via the transfer from $L$ to $K$. The "reduced trace" $\operatorname{trd}_{A / D}$ is defined just before Lemma 1.10.

$\breve{h}: L^{m} \times L^{m} \rightarrow L$ skew symmetric. This means that $\underline{\hat{h}}$ is a skew symmetric matrix (Lemma 1.7), $m$ is even, and $A$ has index 1 . If the involution on $A$ is orthogonal, i.e. $h_{0}^{*}=h_{0}$ so $\epsilon_{0}=1$, then $\hat{h}$ is skew Hermitian (cf. p. 4698), and so $\operatorname{trd}_{L / K} \hat{h}=\frac{g}{n} h$ and $h$ are skew symmetric. Since the skew symmetric case is handled in $₫ 2$, we ignore it here.

If the involution on $A$ is symplectic, $h_{0}$ is skew symmetric and $\hat{h}$ is Hermitian. The transfer $\operatorname{trd}_{A / L} \hat{h}$ is symmetric on $L^{n m}$ with discriminant matrix $-\underline{\hat{h}} \otimes h_{0}$ by (1.7). This implies readily that $\operatorname{trd}_{A / L} \hat{h}$ is hyperbolic, and so $\operatorname{trd}_{A / K} \hat{h}$ and $h$ are also hyperbolic. Thus we get exactly one orthogonal representation, up to equivalence, and it is on a hyperbolic space. This is the index 1 case of (e).

$\breve{h}: L^{m} \times L^{m} \rightarrow L$ symmetric. Thus $\underline{\hat{h}}$ is a symmetric matrix. If the involution on $A$ is symplectic, $\hat{h}$ is skew Hermitian and $h$ is skew symmetric, and this is already handled elsewhere.

Now suppose the involution on $A$ is orthogonal. Then $\hat{h}$ is Hermitian and the transfer $\operatorname{trd}_{A / L} \hat{h}$ is a symmetric form on $L^{n m}$ with discriminant matrix $\underline{\hat{h}} \otimes h_{0}$ and determinant $(\operatorname{det} \underline{\hat{h}})^{n}\left(\operatorname{det} h_{0}\right)^{m}(\operatorname{cf}$. Lemma 1.10), and $h$ is symmetric as well.

Consider first the case $n$ odd. Then the determinant of $b=\operatorname{trd}_{A / L} \hat{h}$ is

$$
(\operatorname{det} \underline{\hat{h}})\left(\operatorname{det} h_{0}\right)^{m}
$$

and so is arbitrary since $\operatorname{det}(\underline{\hat{h}})=\operatorname{det} \breve{h}$ is arbitrary. Assume also that $m>1$. Then by Lemma 1.3 (iv), the Hasse symbol of $b$ is also arbitrary; in other words, $b$ runs over all equivalence classes of symmetric forms on $L^{n m}$ as $\breve{h}$ runs over all equivalence classes of symmetric forms on $L^{m}$ - thus the correspondence is bijective. Now consider the transfer $b_{K}=\operatorname{trd}_{L / K} b$. Since $L / K$ is Abelian,

$$
l=[L: K]=\left[K^{*}: \mathrm{N}_{L / K} L^{*}\right]
$$

by local class field theory, and it follows that, when $l$ is odd, the map

$$
\overline{\mathrm{N}}: L^{*} / L^{* 2} \rightarrow K^{*} / K^{* 2}
$$

induced by the norm is onto; thus it is an isomorphism when $K$ is nondyadic, and in general has kernel of order $\sigma_{2}\left(L^{*}\right) / \sigma_{2}\left(K^{*}\right)$. This means there are exactly 
$\sigma_{2}\left(L^{*}\right) / \sigma_{2}\left(K^{*}\right)$ equivalence classes of orthogonal representations for each symmetric form on $K^{\operatorname{lnm}}$ by Lemmas 1.1 and1.2. This gives (f) when $l$ is odd.

Suppose that $l$ is even. The image of $\overline{\mathrm{N}}$ is $\left(\mathrm{N}_{L / K} L^{*}\right) K^{* 2} / K^{* 2}$, and by local class field theory, $\left(\mathrm{N}_{L / K} L^{*}\right) K^{* 2}$ consists of the norms from the compositum of the quadratic extensions of $K$ contained in $L$; the degree of this compositum over $K$ is $\sigma_{2}(\mathcal{G})=\left(\operatorname{Gal}(L / K): \operatorname{Gal}(L / K)^{2}\right)$. Thus since $\left(L^{*}: L^{* 2}\right)=|\operatorname{imN}| \cdot|\operatorname{ker} \bar{N}|$, each $b_{K}$ is, up to equivalence, the image of $|\operatorname{ker} \overline{\mathrm{N}}|$ of the $b$, where

$$
|\operatorname{ker} \overline{\mathrm{N}}|=\sigma_{2}\left(L^{*}\right) \sigma_{2}(\mathcal{G}) / \sigma_{2}\left(K^{*}\right)
$$

Thus in this case the symmetric forms on $K^{l m n}$ which support an orthogonal representation of $G$ which is linearly equivalent to $\rho$ are those with determinant in $(\operatorname{disc}(L / K))^{m n}\left(\mathrm{~N}_{L / K} L\right)^{*} K^{* 2}$ (since $(g / n)^{l m n}$ is a square), and each of them supports $|\operatorname{ker} \overline{\mathrm{N}}|$ different orthogonal equivalence classes of orthogonal representations. This completes the proof of (f).

Now suppose that $n$ is odd and $m=1$. If $\underline{\hat{h}}=(\alpha), b=\operatorname{trd}_{A / L} \hat{h}=\left\langle\alpha h_{0}\right\rangle$, which has determinant $\alpha^{n} \operatorname{det} h_{0}$. Since $n$ is odd, we get one form $b$ over $L$ for each possible discriminant $\alpha \in L^{*} / L^{* 2}$. By the same analysis as in the case $m>1$, the discriminants which occur among the transfers $b_{K}$ down to $K$ are $(\operatorname{disc}(L / K))\left(\mathrm{N}_{L / K} L^{*}\right) K^{* 2}$ and each discriminant receives $\frac{\sigma_{2}(\mathbf{G}) \sigma_{2}\left(L^{*}\right)}{\sigma_{2}\left(K^{*}\right)}$ of the $b$. This proves $(\mathrm{g})$. We note also that this shows that the possible discriminants of $h$ are $\left(\frac{n}{g}\right)^{l}(\operatorname{disc}(L / K))\left(\mathrm{N}_{L / K} L^{*}\right) K^{* 2}$, as mentioned in Remark 2 after the theorem.

Now consider the case when $n$ is even (and the involution of $A$ is orthogonal). The determinant of the transfer $b=\operatorname{trd}_{A / L} \hat{h}$ down to $L$ is the same for all $\breve{h}$, namely $\left(\operatorname{det} h_{0}\right)^{m}$, and

$$
\mathrm{S}(b)=\mathrm{S}\left(\underline{\hat{h}} \otimes h_{0}\right)=\mathrm{S}\left(h_{0}\right)^{m}\left(\operatorname{det} \underline{\hat{h}}, \operatorname{disc} h_{0}\right)_{L}\left(\operatorname{det} h_{0},-1\right)_{L}^{m(m-1) / 2}
$$

by Lemma 1.3 (iv). If $\operatorname{disc} h_{0} \notin L^{* 2}$, this implies that we get both forms of determinant $\left(\operatorname{det} h_{0}\right)^{m}$ over $L$, and so, by Lemmas 1.1 and 1.2, both forms of determinant $(\operatorname{disc}(L / K))^{m n} \mathrm{~N}_{L / K}\left(\operatorname{det} h_{0}\right)^{m}=\mathrm{N}_{L / K}\left(\operatorname{det} h_{0}\right)^{m}$ over $K$ via the transfer from $L$ to $K$. Furthermore, by (4.3) one of the forms arises from those $\breve{h}$ whose determinants are in the kernel of canonical homomorphism

$$
L^{*} / L^{* 2} \rightarrow L^{*} / \mathrm{N}_{L\left(\sqrt{\operatorname{disc} h_{0}}\right) / L} L\left(\sqrt{\operatorname{disc} h_{0}}\right)^{*} \cong \mathbb{Z} / 2
$$

so the number of equivalence classes of orthogonal representations for each of the two forms is $\sigma_{2}\left(L^{*}\right)$ if $m>1, \frac{1}{2} \sigma_{2}\left(L^{*}\right)$ if $m=1$. This is (h).

On the other hand, disc $h_{0} \in L^{* 2}$ means that $\operatorname{det} h_{0}=(-1)^{n(n-1) / 2}=(-1)^{n / 2}$ and $\operatorname{det} b=(-1)^{m n / 2}$; in this case we get only one form over $L$ as a transfer - that with determinant $(-1)^{m n / 2}$ and Hasse symbol

$$
\mathrm{S}(b)=\mathrm{S}\left(h_{0}\right)^{m}(-1,-1)_{L}^{m n(m-1) / 4}
$$

Therefore there we get only one $G$-invariant symmetric form $b_{K}$ over $K$. Its determinant is $(\operatorname{disc}(L / K))^{m n} \mathrm{~N}_{L / K}(-1)^{m n / 2}=(-1)^{l m n / 2}$, and we now determine its equivalence class. 
Suppose first that $m n / 2$ is even. Then $\operatorname{det} b=1$ and $\operatorname{dim} b=m n \equiv 0 \bmod 4$, so $b \sim_{W} 0$ or $b \sim_{W} g_{L}$. Moreover, by Lemma 1.3)(v),

$$
\begin{aligned}
b \sim_{W} 0 & \Leftrightarrow \mathrm{S}(b)=(-1,-1)_{L}^{m n / 4} \\
& \Leftrightarrow \mathrm{S}\left(h_{0}\right)^{m}(-1,-1)_{L}^{m n(m-1) / 4}=(-1,-1)_{L}^{m n / 4} \\
& \Leftrightarrow \mathrm{S}\left(h_{0}\right)^{m}=(-1,-1)_{L}^{m^{2} n / 4} .
\end{aligned}
$$

On the other hand, if $m n / 2$ is odd, then $\operatorname{det} b=-1$ and $\operatorname{dim} b \equiv 2 \bmod 4$, so if $c$ is a hyperbolic plane, $\operatorname{det}(b \perp c)=1$ and $\operatorname{dim}(b \perp c) \equiv 0 \bmod 4$, and so since $b \sim_{W} b \perp c$, we see again that $b \sim_{W} 0$ or $b \sim_{W} g_{L}$. Now $\mathrm{S}(b \perp c)=\mathrm{S}(b)(-1,-1)_{L}$ by Lemma 1.3 (ii), and by applying the same kind of argument to $b \perp c$ as we did above to $b$, we get (i).

$\breve{h}: L^{m} \times L^{m} \rightarrow L$ skew Hermitian. The involution on $A$ is unitary, so we are assuming that $h_{0}$ is an Hermitian matrix, $h_{0}^{*}=h_{0}$. The form $\hat{h}$ and its discriminant matrix $\underline{\hat{h}}$ are skew Hermitian. If the involution is the identity on $K, h$ is skew symmetric and we can ignore it.

Suppose therefore that the involution is not the identity on $K$, say $K=K_{0}\left(\sqrt{\lambda_{0}}\right)$; then also $L=L_{0}\left(\sqrt{\lambda_{0}}\right)$. The transfer $\operatorname{trd}_{A / L} \hat{h}$ is a skew Hermitian form over $L$ of rank $m n$ and determinant $(\operatorname{det} \underline{\hat{h}})^{n}\left(\operatorname{det} h_{0}\right)^{m}$. Write $\underline{\hat{h}}=\sqrt{\lambda_{0}} \underline{\hat{h}}_{1}$, where $\underline{\hat{h}}_{1}$ is an Hermitian matrix. Then the determinant of $\operatorname{trd}_{A / L} \hat{h}$ is ${\sqrt{\lambda_{0}}}^{m n}\left(\operatorname{det} \underline{\hat{h}}_{1}\right)^{n}\left(\operatorname{det} h_{0}\right)^{m}$, and $\operatorname{det} \underline{\hat{h}}_{1}$ and $\operatorname{det} h_{0}$ are both in $L_{0}^{*}$.

If $n$ is even, we get but one form (up to equivalence) as a transfer over $L$ - it has determinant $\left(\lambda_{0}^{\frac{n}{2}} \operatorname{det} h_{0}\right)^{m}$ - and so only one transfer down to $K$, of determinant $\mathrm{N}_{L / K}\left(\lambda_{0}^{\frac{n}{2}} \operatorname{det} h_{0}\right)^{m}$ by Lemma 1.1 This form gives rise, then, to two inequivalent unitary representations with respect to this unique skew Hermitian form. This is the skew Hermitian version of (b) (cf. the definition of disc $h$ in $\$ 1.1$ ).

Suppose now that $n$ is odd. Then there are two transfers (up to equivalence) down to $L$, with their determinants representing the two possible classes in $L^{*} / \mathrm{N}_{L / L_{0}} L^{*}$. Since the restriction of $\mathrm{N}_{L / K}$ to $L_{0}$ is $\mathrm{N}_{L_{0} / K_{0}}$, the following lemma and Lemma 1.1 imply that their transfers down to $K$ are also distinct, and so we have 2 distinct skew Hermitian forms over $K$ each with a single unitary representation. This is the skew Hermitian version of (a).

Lemma 4.3. The homomorphism $\overline{\mathrm{N}}: L_{0}^{*} / \mathrm{N}_{L / L_{0}} L^{*} \rightarrow K_{0}^{*} / \mathrm{N}_{K / K_{0}} K^{*}$ induced by $\mathrm{N}_{L_{0} / K_{0}}$ is onto.

Proof. Let $\mathrm{Gal}_{L_{0}}^{a b}$ be the Galois group of the Abelian closure $L_{0}^{a b}$ of $L_{0}$ in some separable closure. Define $\mathrm{Gal}_{K_{0}}^{a b}$ similarly, using the same separable closure. Let $\mathrm{Gal}_{L_{0}}$ and $\mathrm{Gal}_{K_{0}}$ be the absolute Galois groups of $L_{0}$ and $K_{0}$. We may assume $\mathrm{Gal}_{L_{0}} \subset \mathrm{Gal}_{K_{0}}$, and this inclusion induces a homomorphism

$$
\mathrm{Gal}_{L_{0}}^{a b}=\mathrm{Gal}_{L_{0}} / \mathrm{Gal}_{L_{0}}^{\prime} \stackrel{i}{\rightarrow} \mathrm{Gal}_{K_{0}}^{a b}=\mathrm{Gal}_{K_{0}} / \mathrm{Gal}_{K_{0}}^{\prime}, \sigma \mathrm{Gal}_{L_{0}}^{\prime} \mapsto \sigma \mathrm{Gal}_{K_{0}}^{\prime}
$$

This fits into the commutative diagram

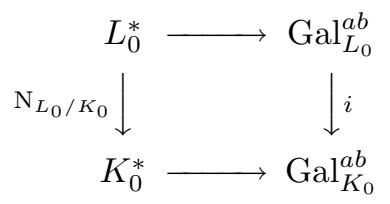


where the horizontal maps are given by the norm residue symbol - cf. [2], p. 141 . This implies a commutative diagram

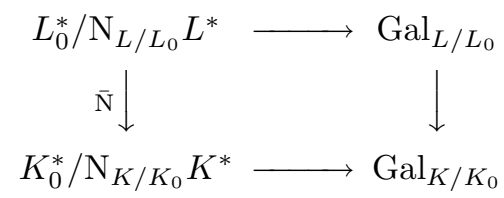

where the right vertical map is restriction - and is an isomorphism. Since the horizontal maps are isomorphisms by local class field theory, $\overline{\mathrm{N}}$ is an isomorphism as well.

$\breve{h}: L^{m} \times L^{m} \rightarrow L$ Hermitian. Again $h_{0}$ is Hermitian and the involution is unitary. The transfer $\operatorname{trd}_{A / L} \hat{h}$ of $\hat{h}$ down to $L$ is Hermitian with determinant $(\operatorname{det} \underline{\hat{h}})^{n}\left(\operatorname{det} h_{0}\right)^{m}$. Thus if $n$ is even we get only one form over $L$ (up to equivalence), while if $n$ is odd we get two.

We consider first the case when the involution is the identity on $K$ (so $h$ is symmetric) and $n$ is even. Thus $\operatorname{trd}_{A / L} \hat{h}$ has determinant $\left(\operatorname{det} h_{0}\right)^{m}$. The transfer of a rank 1 Hermitian form with matrix $(\eta)$ over $L=L_{0}\left(\sqrt{\lambda_{0}}\right)$ down to $L_{0}$ is the symmetric form $\left\langle 2 \eta,-2 \eta \lambda_{0}\right\rangle$, so the transfer of $\hat{h}$ down to $L_{0}$ is the symmetric form of rank $2 m n$ with matrix $\operatorname{trd}_{A / L}\left(\hat{h} \otimes\left\langle 2,-2 \lambda_{0}\right\rangle\right)$, under the assumption that $\operatorname{trd}_{A / L} \hat{h}$ is diagonal - in fact we can assume that $\operatorname{trd}_{A / L} \hat{h}=\left\langle 1, \ldots, 1,\left(\operatorname{det} h_{0}\right)^{m}\right\rangle$. The determinant of this form over $L_{0}$ is

$$
\left(\operatorname{det} \operatorname{trd}_{A / L} \hat{h}\right)^{2}\left(-\lambda_{0}\right)^{m n}=1,
$$

and by Lemma 1.3 (iv) its Hasse invariant is

$$
(-1,-1)_{L_{0}}^{m n / 2}\left((-1)^{n / 2} \operatorname{det} h_{0}, \lambda_{0}\right)_{L_{0}}^{m}
$$

Since its rank is divisible by 4 , it is $\sim_{W} 0$ or $g_{L_{0}}$, and is $\sim_{W} 0$ if and only if $(-1,-1)_{L_{0}}^{m n / 2}\left((-1)^{n / 2} \operatorname{det} h_{0}, \lambda_{0}\right)_{L_{0}}^{m}=(-1,-1)_{L_{0}}^{m n / 2}$ by Lemma 1.3. this condition is equivalent to $(-1)^{m n / 2}\left(\operatorname{det} h_{0}\right)^{m} \in \mathrm{N}_{L / L_{0}} L^{*}$. By Lemma 1.2, the same situation obtains when we take the transfer down to $K$, and in either case we get a single symmetric form $h$ supporting 2 nonequivalent orthogonal representations. This is (d).

Now suppose that the involution is the identity on $K$ and $n$ is odd. As in the case $n$ even, the two transfers to $L_{0}$ have matrix $\left(\operatorname{trd}_{A / L} \hat{h}\right) \otimes\left\langle 2,-2 \lambda_{0}\right\rangle$. Their determinants are both $\left(-\lambda_{0}\right)^{m n}=\left(-\operatorname{disc}\left(L / L_{0}\right)\right)^{m}$, and their Hasse invariants are given by

$$
\left((-1)^{m(m n-1) / 2}\left(2 \operatorname{det} h_{0}\right)^{m} \operatorname{det} \underline{\hat{h}}, \lambda_{0}\right)_{L_{0}}(-1,-1)_{L_{0}}^{m(m n-1) / 2} .
$$

These Hasse invariants are distinct since $\operatorname{det} \underline{\hat{h}}$ represents both norm residue classes in $L_{0}^{*} / \mathrm{N}_{L / L_{0}} L^{*}$, and so the transfers to $L_{0}$ remain distinct. Both transfers to $K$ have determinant $\mathrm{N}_{L_{0} / K}\left(-\operatorname{disc}\left(L / L_{0}\right)\right)^{m}$, and they remain inequivalent by Lemma 1.2. This is (c).

Now suppose the involution is not the identity on $K$. As before, the transfer $\operatorname{trd}_{A / L} \hat{h}$ is an Hermitian form over $L$ with determinant $(\operatorname{det} \hat{h})^{n}\left(\operatorname{det} h_{0}\right)^{m}$. If $n$ is even, we get but one form (up to equivalence) as a transfer over $L$ - it has determinant $\left(\operatorname{det} h_{0}\right)^{m}$ and rank $m n$ - and so also only one transfer down to $K$, of determinant $\mathrm{N}_{L_{0} / K_{0}}\left(\operatorname{det} h_{0}\right)^{m}$ by Lemma 1.1 and rank $l m n$. Thus this form 
supports two inequivalent unitary representations. This is (b) in the Hermitian case.

If $n$ is odd, there are two distinct transfers (up to equivalence) down to $L$, with determinants $\operatorname{det} \hat{h}\left(\operatorname{det} h_{0}\right)^{m}$ representing the two norm classes in $L_{0}^{*} / \mathrm{N}_{L / L_{0}} L^{*}$. By Lemmas 1.1 and 4.3. their transfers down to $K$ are also inequivalent, and so we have two inequivalent Hermitian forms over $K$, each with a single unitary representation. This is (a) in the Hermitian case.

The remaining cases involve $A$ of index $>1$. Thus $A=\mathrm{M}(n, D)$, where $D$ is a quaternion division algebra over $K$ with conjugation * as its involution. Since an Hermitian or skew Hermitian form over $D$ has an orthogonal basis, we can suppose that $h_{0}$ is diagonal, say $h_{0}=\left\langle\eta_{01}, \ldots, \eta_{0 n}\right\rangle$, where $\eta_{0 j}^{*}=\epsilon_{0} \eta_{0 j}$ for all $j$. If $\epsilon_{0}=1$, we can assume that $h_{0}=\mathrm{I}_{n}$ (cf. Table 1$)$.

Similarly $\breve{h}$ is either Hermitian or skew Hermitian, and we can assume that its matrix $\epsilon_{0} \underline{\hat{h}}$ is diagonalized, say $\underline{\hat{h}}=\left\langle\eta_{1}, \ldots, \eta_{m}\right\rangle$, and that $\underline{\hat{h}}=\mathrm{I}_{m}$ if $\breve{h}$ is Hermitian.

$\breve{h}: D^{m} \times D^{m} \rightarrow D$ Hermitian. Suppose first that $h_{0}$ is $\operatorname{Hermitian}$ (i.e. $\left(A,{ }^{-}\right)$ is symplectic of index $>1-$ cf. $\underline{1.2}$, so $h_{0}=\mathrm{I}_{n}, \underline{\hat{h}}=\mathrm{I}_{m}$, and $\hat{h}(u, v)=u v^{*}$. By Lemma 1.11, (b), if we define the "trace" $\operatorname{trd}_{A / D}\left(a_{i j}\right)=\sum_{i} a_{i i}$, then $\operatorname{trd}_{A / D} \hat{h}$ is the nonsingular Hermitian form on $D^{n m}$ with respect to conjugation, with discriminant matrix $\mathrm{I}_{n m}$. Furthermore $\operatorname{trd}_{A / L} \hat{h}=\operatorname{trd}_{D / L} \operatorname{trd}_{A / D} \hat{h}$, and so it follows that $\operatorname{trd}_{A / L} \hat{h}$ is the orthogonal direct sum of $n m$ copies of the norm form $g_{L}$ of $D$. Since $g_{L} \perp g_{L}$ is hyperbolic, $\operatorname{trd}_{A / L} \hat{h}$ is hyperbolic of rank $4 n m$ if $n$ or $m$ is even - in which case $\operatorname{trd}_{A / K} \hat{h}$ is also hyperbolic, of rank $4 \operatorname{lnm}$. Otherwise $\operatorname{trd}_{A / L} \hat{h} \sim_{W} g_{L}$, and by Lemma 1.2 $\operatorname{trd}_{A / K} \hat{h} \sim_{W} g_{K}$.

In each of these two cases, $\mathrm{nm}$ even or odd, we get a unique orthogonal representation linearly equivalent to $\rho$. This is (e) in the nonsplit case.

If $h_{0}$ is skew Hermitian $\left(\left(A,{ }^{-}\right)\right.$is orthogonal with index $\left.>1\right)$, then $\hat{h}$ is also skew Hermitian. It follows that $\operatorname{trd}_{A / K} \hat{h}$ is skew symmetric, so we can ignore it.

$\breve{h}: D^{m} \times D^{m} \rightarrow D$ skew Hermitian. If $h_{0}$ is Hermitian $\left(\left(A,{ }^{-}\right)\right.$symplectic with index $>1)$, then $\hat{h}$ is skew Hermitian and the transfer $\operatorname{trd}_{A / K} \hat{h}$ is a skew symmetric form (on $\left.K^{4 \operatorname{lnm}}\right)$.

Suppose then that $\left(A,{ }^{-}\right)$is orthogonal, so $h_{0}$ is skew Hermitian, $\hat{h}$ is Hermitian and $\underline{\hat{h}}$ is a skew Hermitian matrix. By Lemma $1.11(\mathrm{a}), \operatorname{trd}_{A / L} \hat{h}$ is the orthogonal direct sum of the $m n$ transfers

$$
h_{i j}(u, v)=\operatorname{trd}_{D / L}\left(u \eta_{i} v^{*} \eta_{0 j}\right) \quad(1 \leq i \leq m, 1 \leq j \leq n)
$$

of one dimensional forms over $D$. By Lemma 1.12, $h_{i j}$ is a quaternary symmetric form of determinant 1 , and is hyperbolic if and only if $\left(\eta_{i}^{2}, \eta_{0 j}^{2}\right)_{L}=-1$. It follows that $\operatorname{trd}_{A / L} \hat{h} \sim_{W} 0$ if and only if

$$
\prod_{i, j}\left(\eta_{i}^{2}, \eta_{0 j}^{2}\right)_{L}=(-1)^{m n}
$$

and otherwise it is $\sim_{W} g_{L}$. Now

$$
\prod_{i} \eta_{i}^{2}=\prod_{i}\left(-\eta_{i} \eta_{i}^{*}\right)=\prod_{i}\left(-\operatorname{nrd}_{D / L} \eta_{i}\right)=(-1)^{m} \operatorname{nrd}_{B / L} \underline{\hat{h}}
$$

$(B=\mathrm{M}(m, D))$, so $\operatorname{trd}_{A / L} \hat{h} \sim_{W} 0$ if and only if

$$
\left((-1)^{m} \operatorname{nrd}_{B / L} \underline{\hat{h}},(-1)^{n} \operatorname{nrd}_{A / L} h_{0}\right)_{L}=(-1)^{m n} .
$$


Parts $(\mathrm{j})$ and $(\mathrm{k})$ now follow if one takes into account the following facts, along with Lemma 1.2.

(i) Two nonsingular skew Hermitian forms of the same rank over $D$ are equivalent if and only if their determinants are equal $\left(\right.$ in $\left.L^{*} / L^{* 2}\right)$.

(ii) For any fixed rank $r$ and square class $\lambda \in L^{*} / L^{* 2}$, there is a skew Hermitian form of rank $r$ and determinant $\lambda$ unless $r=1$ and $\lambda=-1$. See [21], Theorem 3.6, p. 363 .

This finishes the proof of Theorem 4.1

Corollary 4.4. We use the same notation and assumptions as in Theorem 4.1; in particular $K$ is a local non-Archimedean field and $\rho$ is isotypic. Assume in addition that $\rho$ has odd type.

Unitary representations. There is exactly 1 equivalence class of unitary representations $G \rightarrow \mathbf{U}(V, h)$ linearly equivalent to $\rho$.

Thus two unitary representations of odd type are equivalent if and only if they are linearly equivalent and their underlying forms are isotypically equivalent.

Orthogonal representations. The number of equivalence classes of orthogonal representations $G \rightarrow \mathbf{O}(V, h)$ which are linearly equivalent to $\rho$ is as follows:

$\begin{array}{ccc}\begin{array}{c}\text { kind of } \\ \left(A,{ }^{-}\right)\end{array} & \begin{array}{c}\text { \# of equiv. } \\ \text { classes }\end{array} & \text { conditions } \\ \text { first } & \frac{\sigma_{2}(\mathcal{G}) \sigma_{2}\left(L^{*}\right)}{\sigma_{2}\left(K^{*}\right)} & m>1 \text { and } \operatorname{det} h \in(\operatorname{disc}(L / K))^{m}\left(\mathrm{~N}_{L / K} L^{*}\right) K^{* 2}, \\ \text { first } & 1 \leq \# & m=1 \text { and } h \cong \operatorname{Tr}_{L / K}\left\langle\alpha h_{0}\right\rangle, \alpha \in L^{*} \\ & \leq \frac{\sigma_{2}(\mathcal{G}) \sigma_{2}\left(L^{*}\right)}{\sigma_{2}\left(K^{*}\right)} & \\ \text { second } & 1 & \operatorname{det} h=\mathrm{N}_{L_{0} / K}\left(-\operatorname{disc}\left(L / L_{0}\right)\right)^{m}\end{array}$

and 0 otherwise.

When $G$ is Abelian, we get

kind of $\left(A,{ }^{-}\right) \quad$ \# of equiv. classes conditions

$\begin{array}{ccc}\text { first } & 1 \\ \text { second } & 1 & \operatorname{det} h=\mathrm{N}_{L_{0} / K}\left(-\operatorname{disc}\left(L / L_{0}\right)\right)^{m},\end{array}$

and 0 otherwise.

Two not necessarily isotypic orthogonal representations of odd type, which we assume to be split if $G$ is not Abelian, are equivalent if and only if they are linearly equivalent and their underlying forms are isotypically equivalent.

Remark 4.5. When $G$ is Abelian and the involution on $K$ is the identity, the restriction of the involution of $K G$ to any direct summand $L$ must be nontrivial if $L \neq K$ (i.e the involution is unitary), since $L$ is generated over $K$ by the images of $G$ under the projection $K G \rightarrow L$.

Proof. The assumption that $\rho$ has odd type means that $A$ has index 1 , and that $n$ is odd. The statements about the number of equivalence classes in each case follow by Theorem 4.1 - and by Remark 4.5 in the case of an orthogonal representation of an Abelian group.

The statements about the equivalence of two not necessarily isotypic representations follow since the conditions stated for orthogonal representations rule out the cases where the number of equivalence classes of isotypic representations is $>1$.

We now treat in detail the nondyadic case in Theorem 4.1, (g), for orthogonal representations, and we begin with two auxiliary lemmas. We assume that $K_{\mathrm{nr}}$ is 
the unramified closure of $K$ in $L$, with $\left(K_{\mathrm{nr}}: K\right)=f$, and that $K_{\mathrm{tm}}$ is the largest tamely ramified subextension of $L / K$, with $\left(K_{\mathrm{tm}}: K_{\mathrm{nr}}\right)=e$. Let $\left(L: K_{\mathrm{tm}}\right)=q$, which is a power of the residue class field characteristic $p$ and so is odd.

Recall that $\mathcal{G}=\operatorname{Gal}(L / K)$. Note that in the case when $K$ is nondyadic, $\sigma_{2}(\mathcal{G})=$ $\left(\mathcal{G}: \mathcal{G}^{2}\right)=1,2$, or 4 (since, for example, it is the order of the kernel of the map $L^{*} / L^{* 2} \rightarrow K^{*} / K^{* 2}$ induced by the norm, according to the proof of (f) of Theorem 4.1).

Lemma 4.6. (a) The Galois group $\mathrm{Gal}\left(K_{\mathrm{tm}} / K\right)$ is isomorphic to the direct product of $\operatorname{Gal}\left(K_{\mathrm{nr}} / K\right)$ and $\operatorname{Gal}\left(K_{\mathrm{tm}} / K_{\mathrm{nr}}\right)$.

(b) If $K$ is nondyadic or $L / K$ is tamely ramified, then

$$
\sigma_{2}(\mathcal{G})= \begin{cases}1 & \text { if and only if } e \text { and } f \text { are odd, } \\ 2 & \text { if and only if } e \text { and } f \text { have different parity, } \\ 4 & \text { if and only if } e \text { and } f \text { are both even. }\end{cases}
$$

Proof. (a) is a special case of [18, aufgabe 1, p. 1851, according to which

$$
\operatorname{Gal}(L / K) \cong \operatorname{Gal}\left(L / K_{\mathrm{nr}}\right) \rtimes \operatorname{Gal}\left(K_{\mathrm{nr}} / K\right)
$$

for any tamely ramified Galois extension $L / K$ of a Henselian field $K$. (b) follows at once from (a), since the wild ramification index $\left(L: K_{\mathrm{tm}}\right)$ is odd.

Let $c_{K}(h)$ denote the Witt invariant of the symmetric form $h$. The following lemma is easily checked.

Lemma 4.7. (a) If the rank of $h$ is odd, then $c_{K}(\alpha h)=c_{K}(h)$ for any $\alpha \in K^{*}$.

(b) If the rank of $h$ is even and $K$ is nondyadic, then $c_{K}(\alpha h)=c_{K}(h)$ for any unit $\alpha \in K^{*}$.

If $M / K$ is any finite separable extension, $\langle M\rangle$ stands for the symmetric form $\operatorname{Tr}_{M / K}(x y)(x, y \in M)$; in other, words, $\langle M\rangle$ is the transfer of the symmetric bilinear form over $M$ with matrix (1). We also denote by $\operatorname{ord}_{K} \alpha$ the order of $\alpha \in K^{*}$ with respect to a prime element of the $K$.

Let $h$ be a symmetric form of rank $n$ over $L$. Define

$$
\begin{aligned}
r & =\operatorname{ord}_{K_{\mathrm{tm}}} \operatorname{disc}\left(\operatorname{Tr}_{L / K_{\mathrm{tm}}} h\right) \\
& =\operatorname{ord}_{K_{\mathrm{tm}}}\left(\left(\operatorname{disc} L / K_{\mathrm{tm}}\right)^{n} \mathrm{~N}_{L / K_{\mathrm{tm}}}(\operatorname{disc} h)\right) \\
& =n \operatorname{ord}_{K_{\mathrm{tm}}}\left(\operatorname{disc} L / K_{\mathrm{tm}}\right)+\operatorname{ord}_{L}(\operatorname{disc} h) .
\end{aligned}
$$

Lemma 4.8. Suppose that $K$ is a nondyadic field and that the rank $n$ of $h$ is odd.

(a) If $(L: K)$ is odd,

$$
\mathrm{c}_{K}\left(\operatorname{Tr}_{L / K} h\right)=\mathrm{c}_{L} h
$$

(b) If $(L: K)$ is even,

$$
\mathrm{c}_{K}\left(\operatorname{Tr}_{L / K} h\right)=\mathrm{c}_{K}\left\langle K_{\mathrm{tm}}\right\rangle^{r+1}\left(\pi, \operatorname{disc}\left(\operatorname{Tr}_{L / K} h\right)\right)_{K}^{e r}\left(\Pi, \operatorname{disc}\left(\operatorname{Tr}_{L / K_{\mathrm{tm}}} h\right)\right)_{K_{\mathrm{tm}}}^{(e+1)(r+1)} \mathrm{c}_{L} h,
$$

and

$$
\mathrm{c}_{K}\left\langle K_{\mathrm{tm}}\right\rangle=\left\{\begin{array}{cl}
1 & \text { if } e \text { is odd } \\
\left(\pi,(-1)^{(f-1) / 2} e\right)_{K} & \text { if } e \text { is even and } f \text { is odd } \\
-\left(\pi,(-1)^{f / 2} \operatorname{disc} L / K\right)_{K} & \text { if } e \text { and } f \text { are both even }
\end{array}\right.
$$

\footnotetext{
${ }^{1}$ I am grateful to M. Kolster for providing this reference.
} 
Here $\pi$ and $\Pi$ are prime elements of $K$ and $K_{\mathrm{tm}}$ respectively, arbitrary unless $f$ is odd, in which case they are chosen to satisfy $\Pi^{e} \equiv-\pi \bmod K_{\mathrm{nr}}^{* 2}$.

Proof. The first formula (4.4) follows from [5], Theorem 4.4, 3. The second formula follows from [5], Theorem 5.6, after a straightforward computation. It uses the facts that if $\alpha \in L$, then $(\pi, \alpha)_{L}=\left(\pi, \mathrm{N}_{L / K} \alpha\right)_{K}$ (cf. [12], Theorem 2.14, 8.), and that $\mathrm{c}_{K_{\mathrm{tm}}}\left(\operatorname{Tr}_{L / K_{\mathrm{tm}}} h\right)=\mathrm{c}_{L} h$ (which is the special case of (4.4) with $K=K_{\mathrm{tm}}$ ). The formula for $\mathrm{c}_{K}\left\langle K_{\mathrm{tm}}\right\rangle$ follows from [5], Theorems 4.2, 5.3, 5.4, and 5.5.

Theorem 4.9. Let $K$ be a nondyadic local field, and suppose that $\left(A,{ }^{-}\right)$is orthogonal of odd degree $n$ with center $L$ and index 1 . We denote by $f, e$, and $q$ respectively the index of inertia, the tame ramification index, and the wild ramification index of $L / K$.

Suppose that $\rho$ is a linear representation of type $A$ on the vector space $V$ of length 1 , and that $h$ is a symmetric form on $V$.

1. $e$ and $f$ odd. Then there is an invariant form equivalent to $h$ if and only if $c_{K}(h)=c_{L}\left(h_{0}\right)$, and each invariant form admits exactly one orthogonal representation (up to equivalence, of course).

2. e odd and $f$ even. An invariant form has determinant $\operatorname{disc} L / K$ or $\epsilon \operatorname{disc} L / K$, where $\epsilon$ is any nonsquare unit of $K$. Assume that $h$ has one of these as its determinant.

If $(\pi \text {, disc } h)_{K}=1$, there is a form equivalent to $h$ which supports two nonequivalent orthogonal representations if $\mathrm{c}_{K}(h)=\mathrm{c}_{L}\left(h_{0}\right)$; otherwise there is no invariant form equivalent to $h$.

If $(\pi \text {, disc } h)_{K}=-1$, there is an invariant form equivalent to $h$ and it supports one orthogonal representation.

3. e even and $f$ odd. There is an invariant form equivalent to $h$ if and only if $\operatorname{det} h \in(\operatorname{disc}(L / K))\left(\mathrm{N}_{L / K} L^{*}\right) K^{* 2}$ and, if $\operatorname{ord}_{K}(\operatorname{det} h)$ is even, $\mathrm{c}_{K}(h)=$ $\mathrm{c}_{L}\left(h_{0}\right)$. In the latter case, an invariant form supports two inequivalent orthogonal representations. Each of the two inequivalent invariant forms with determinant of odd order supports a single orthogonal representation.

4. $e$ and $f$ both even. There is an invariant form equivalent to $h$ if and only if $\operatorname{det} h=\operatorname{disc}(L / K)$, and it admits three inequivalent orthogonal representations if $c_{K}(h)=c_{L}\left(h_{0}\right)$, and one otherwise.

Proof. We know that the invariant forms on $V$ are those of the form $\operatorname{Tr}_{L / K} \alpha h_{0}$ for $\alpha \in L^{*}$. Since $n$ is odd, we can assume that $\operatorname{det} h_{0}=1$. Then $\operatorname{det} \operatorname{Tr}_{L / K} \alpha h_{0}=$ $\operatorname{disc}(L / K) \mathrm{N}_{L / K} \alpha$ by Lemma 1.1

Suppose $e$ and $f$ are both odd. Then $(L: K)$ is also odd, and by (4.4) and Lemma 4.7 (a), $\mathrm{c}_{K} \operatorname{Tr}_{L / K} \alpha h_{0}=\mathrm{c}_{L} h_{0}$. Since the map $L^{*} / L^{* 2} \rightarrow K^{*} / K^{* 2}$ is an isomorphism when $(L: K)$ is odd, the theorem follows in this case.

Suppose $e$ is odd and $f$ is even. We first prove that the image of $\bar{N}: L^{*} / L^{* 2} \rightarrow$ $K^{*} / K^{* 2}$ consists of the square classes $K^{* 2}$ and $\epsilon K^{* 2}$, where $\epsilon$ is any nonsquare unit of $K$. (Note that $\left(U_{K}: U_{K}^{2}\right)=2$ since $K$ is nondyadic.) The image certainly is of order 2, since its kernel has order $\sigma_{2}(\mathcal{G})=2$ by (4.2) and Lemma 4.6. The norm-induced map $L^{*} / L^{* 2} \rightarrow K_{\mathrm{nr}}^{*} / K_{\mathrm{nr}}^{* 2}$ is bijective since the degree of $L / K_{\mathrm{nr}}$ is odd. The norm of a nonsquare unit of $K_{\mathrm{nr}}$ in $K$ is a nonsquare since 


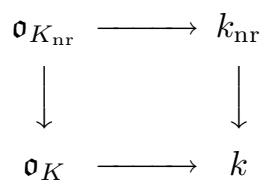

is commutative, and the norm in a finite extension of finite fields is onto. (Here $\mathfrak{o}$ is a ring of integers, $k$ its residue class field, and the vertical maps are norms.) Thus the image of $\bar{N}$ is as stated. It also follows that each of these images is the image of a square class of $L$ containing a unit and of one containing a prime.

Thus $\operatorname{det}\left(\operatorname{Tr}_{L / K} \alpha h_{0}\right)=\operatorname{disc}(L / K) \mathrm{N}_{L / K} \alpha=\operatorname{disc}(L / K)$ or $\epsilon \operatorname{disc}(L / K)$, so an invariant form must have one of these determinants. By Lemma 4.8 (b),

$$
\mathrm{c}_{K}\left(\operatorname{Tr}_{L / K} \alpha h_{0}\right)=\left(\pi, \operatorname{disc}\left(\operatorname{Tr}_{L / K} \alpha h_{0}\right)\right)_{K}^{r} \mathrm{c}_{L}\left(h_{0}\right),
$$

where $r=\operatorname{ord}_{K_{\mathrm{tm}}} \operatorname{disc}\left(L / K_{\mathrm{tm}}\right)+\operatorname{ord}_{L} \alpha$. (We can drop the $n$ from this expression, since $n$ is odd and $r$ only appears in the exponents of powers of -1 .) Thus

$$
\begin{aligned}
\mathrm{c}_{K}\left(\operatorname{Tr}_{L / K} \alpha h_{0}\right) & =\mathrm{c}_{L}\left(h_{0}\right) \quad \text { if } \operatorname{ord}_{L} \alpha \equiv \operatorname{ord} K_{\mathrm{tm}} \operatorname{disc}\left(L / K_{\mathrm{tm}}\right) \bmod 2, \\
& =\left(\pi, \operatorname{disc}\left(\operatorname{Tr}_{L / K} \alpha h_{0}\right)\right)_{K} \mathrm{c}_{L}\left(h_{0}\right) \quad \text { otherwise. }
\end{aligned}
$$

By local class field theory, $\mathrm{N}_{K(\sqrt{\pi}) / K} K(\sqrt{\pi})^{*}$ consists of two of the four square classes in $K^{*} / K^{* 2}$, and it is clear they must be $K^{* 2}$ and $-\pi K^{* 2}$. It follows that $\epsilon$ is not a norm from $K(\sqrt{\pi})$, and so $(\pi, \epsilon)_{K}=-1$. Since an invariant form $h$ satisfies $\mathrm{c}_{K}(h)=\mathrm{c}_{L}\left(h_{0}\right)$ if it arises from an $\alpha$ satisfying $\operatorname{ord}_{L} \alpha \equiv \operatorname{ord}_{K_{\mathrm{tm}}} \operatorname{disc}\left(L / K_{\mathrm{tm}}\right) \bmod 2$, and otherwise satisfies $\mathrm{c}_{K}(h)=(\pi, \operatorname{disc}(h))_{K} \mathrm{c}_{L}\left(h_{0}\right)$, the statement in the theorem for $e$ odd and $f$ even follows readily.

Suppose next that $e$ is even and $f$ is odd. The norm from $L$ to $K_{\text {tm }}$ (on nonzero elements) is bijective mod squares since the degree is odd. It follows from Lemma 4.6. (b), that the norm from $K_{\mathrm{tm}}$ to $K_{\mathrm{nr}}$ takes units to square units, and all primes to a single square class containing primes, and so the same thing is true for the norm from $L$ to $K$ since $\left(K_{\mathrm{nr}}: K\right)=f$ is odd.

By Lemma 4.8

$$
\mathrm{c}_{K}\left(\operatorname{Tr}_{L / K} \alpha h_{0}\right)=\mathrm{c}_{K}\left\langle K_{\mathrm{tm}}\right\rangle^{r+1}\left(\Pi, \operatorname{disc}\left(\operatorname{Tr}_{L / K_{\mathrm{tm}}} \alpha h_{0}\right)\right)_{K_{\mathrm{tm}}}^{r+1} \mathrm{c}_{L} h_{0} .
$$

Thus if $\operatorname{ord}_{L} \alpha \not \equiv \operatorname{ord}_{K_{\mathrm{tm}}} \operatorname{disc}\left(L / K_{\mathrm{tm}}\right) \bmod 2$, then $\mathrm{c}_{K}\left(\operatorname{Tr}_{L / K} \alpha h_{0}\right)=\mathrm{c}_{L}\left(h_{0}\right)$; since $\operatorname{ord}_{L} \alpha \equiv \operatorname{ord}_{L} \alpha^{\prime} \bmod 2$ implies that the norms of $\alpha$ and $\alpha^{\prime}$ down to $K$ are equal mod squares, we get one invariant form supporting two inequivalent orthogonal representations.

We now show that the condition $\operatorname{ord}_{L} \alpha \not \equiv \operatorname{ord}_{K_{\mathrm{tm}}} \operatorname{disc}\left(L / K_{\mathrm{tm}}\right) \bmod 2$ is equivalent to $\operatorname{ord}_{K}\left(\operatorname{det} \operatorname{Tr}_{L / K} \alpha h_{0}\right)$ being even. Note that

$$
\operatorname{ord}_{L} \alpha \text { and } \operatorname{ord}_{K} \mathrm{~N}_{L / K} \alpha
$$

have the same parity, and the same is true of

$$
\operatorname{ord}_{K_{\mathrm{tm}}} \operatorname{disc}\left(L / K_{\mathrm{tm}}\right) \text { and } \operatorname{ord}_{K} \mathrm{~N}_{K_{\mathrm{tm}} / K} \operatorname{disc}\left(L / K_{\mathrm{tm}}\right) .
$$

Thus the condition is equivalent to

$$
\begin{aligned}
\operatorname{ord}_{K}\left(\operatorname{disc}(L / K) \mathrm{N}_{L / K} \alpha\right) & \not \equiv \operatorname{ord}_{K}\left(\operatorname{disc}(L / K) \mathrm{N}_{K_{\mathrm{tm}} / K} \operatorname{disc}\left(L / K_{\mathrm{tm}}\right)\right) \\
& \equiv \operatorname{ord}_{K}\left(\operatorname{disc}\left(K_{\mathrm{tm}} / K\right)^{q}\left(\mathrm{~N}_{K_{\mathrm{tm}} / K} \operatorname{disc}\left(L / K_{\mathrm{tm}}\right)\right)^{2}\right) \\
& \equiv \operatorname{ord}_{K}\left(\operatorname{disc}\left(K_{\mathrm{tm}} / K\right)\right. \\
& =f(e-1) \quad \text { by Dedekind's discriminant theorem } \\
& \equiv 1 \bmod 2,
\end{aligned}
$$

as desired. 
Now suppose that $\operatorname{ord}_{L} \alpha \equiv \operatorname{ord}_{K_{\mathrm{tm}}} \operatorname{disc}\left(L / K_{\mathrm{tm}}\right) \bmod 2$. By a now familiar argument, $\left(\Pi, \mathrm{N}_{L / K_{\mathrm{tm}}} \alpha\right)_{K_{\mathrm{tm}}}$ takes on both \pm 1 for the two possible square classes that $\alpha$ represents, and so we get two invariant forms, each supporting one orthogonal representation. This finishes the proof of the case $e$ even and $f$ odd.

Now suppose both $e$ and $f$ are even. Then $\operatorname{det} \operatorname{Tr}_{L / K} \alpha h_{0}=\operatorname{disc}(L / K)$ by Lemma 4.6 (b). Also (4.5) again holds, and so, if $\operatorname{ord}_{L} \alpha \not \equiv \operatorname{ord}_{K_{\mathrm{tm}}} \operatorname{disc}\left(L / K_{\mathrm{tm}}\right) \bmod 2$, we get one invariant form of determinant $\operatorname{disc}(L / K)$ and Witt invariant $\mathrm{c}_{L}\left(h_{0}\right)$ with two inequivalent orthogonal representations. In the other case, we get two inequivalent invariant forms, again with determinant $\operatorname{disc}(L / K)$, with one orthogonal representation for each. This finishes the proof of the case $e$ and $f$ both even, and of the theorem as well.

\section{Global fields and the Hasse Principle}

In this section, $K$ is a global field with an involution ${ }^{-}$. Our principal goals are to consider problems I, II and III for split representations over $K$, and to prove the Hasse Principle for equivariant representations.

In the case of split representations, it is particularly easy to determine the equivariant representations of $G$ : if $A$ is simple with involution $\bar{a}=h_{0}^{-1} a^{*} h_{0}$, the association $\underline{\hat{h}} \rightsquigarrow \underline{\hat{h}} \otimes h_{0}$ yields a bijective correspondence between the equivalence classes of $\epsilon$-Hermitian forms of a given rank $m$ and the equivalence classes of all equivariant representations of $G$ of rank $m n$ and type $A$ with respect to $\epsilon_{0} \epsilon$-Hermitian forms. (It is understood that if the involution on $K$ is the identity, then Hermitian means symmetric.) As we already have seen, it is easy to calculate such things as the determinant, Hasse symbol and signatures from this formula.

The case of split symplectic representations has already been covered in \$2, so we deal only with unitary and orthogonal representations.

If $K_{0}$ is formally real and $h_{0}$ is symmetric or Hermitian, we can assume that $h_{0}$ is a positive definite matrix at each ordering of $K_{0}$.

If $h$ is a symmetric bilinear form over $\mathbf{R}$ or an Hermitian form over $\mathbf{C}$, the number of -1 's in any diagonalization $\langle 1, \ldots, 1,-1, \ldots,-1\rangle$ is the negative index of $h$. Similarly the number of 1 's is the positive index. If $h$ is a symmetric or Hermitian form over the number field $K$ and $\mathfrak{p}$ is a real prime of $K_{0}$ which does not split in $K$, the indices of $h_{\mathfrak{p}}$ are denoted by $r_{\mathfrak{p}}^{-}(h)$ and $r_{\mathfrak{p}}^{+}(h)$ respectively.

Lemma 5.1. Let $\delta \in K_{0}^{*}$. Let $\wp$ be the set of all real primes of $K_{0}$ which do not split in $K$. Suppose that $m$ is a positive integer and, for each $\mathfrak{p} \in \wp$, that $r_{\mathfrak{p}}$ is an integer such that $0 \leq r_{\mathfrak{p}} \leq m$ and $\delta(-1)^{r_{\mathfrak{p}}}>_{\mathfrak{p}} 0$. Then there is an $m \times m$ Hermitian matrix $\underline{\hat{h}}$ whose determinant is $\delta$ and whose negative index at each $\mathfrak{p} \in \wp$ is $r_{\mathfrak{p}}$.

Proof. If $m=1$, take $\underline{\hat{h}}=(\delta)$. Suppose $m>1$. By the weak approximation theorem, there is an $\alpha_{m} \in K_{0}^{*}$ such that $\alpha_{m}>_{\mathfrak{p}} 0$ if $r_{\mathfrak{p}}<m, \alpha_{m}<_{\mathfrak{p}} 0$ if $r_{\mathfrak{p}}=m$. Put $\delta^{\prime}=\delta \alpha_{m}, r_{\mathfrak{p}}^{\prime}=r_{\mathfrak{p}}$ if $\alpha_{m}>_{\mathfrak{p}} 0,=r_{\mathfrak{p}}-1$ if $\alpha_{m}<_{\mathfrak{p}} 0$. Then $\delta^{\prime}(-1)^{r_{\mathfrak{p}}^{\prime}}>0$, and so by induction there is an Hermitian matrix $\underline{\hat{h}}^{\prime}$ of rank $m-1$ with $\operatorname{det} \underline{\hat{h}}^{\prime}=\delta^{\prime}$ and with negative indices $r_{\mathfrak{p}}^{\prime}$. Then $\underline{\hat{h}}=\underline{\hat{h}}^{\prime} \perp\left(\alpha_{m}\right)$ has the required properties.

Theorem 5.2 (Split unitary representations). Suppose that the simple involution algebra component $A$ of $K G$ is $\cong \mathrm{M}(n, K)$, and that $K$ is a global field with a nontrivial involution. Let $\wp$ be the set of real primes of $K_{0}$ which do not split in $K$. Let $V$ be an A-module of length $m$ and $h$ an Hermitian form on $V$. 
I. There is an invariant form on $V$ equivalent to $h$ if and only if the positive and negative indices of $h$ are divisible by $n$ for each real prime $\mathfrak{p} \in \wp$ and, when $n$ is even, $\operatorname{det} h=\left(\operatorname{det} h_{0}\right)^{m}$.

II. Suppose $h$ is invariant.

(i) If $n$ is odd, $h$ supports exactly one unitary representation of type $A$

(up to equivalence).

(ii) If $n$ is even, the equivalence classes of unitary representations supported by $h$ are in bijective correspondence with the norm classes in $K_{0}^{*} / \mathrm{N}_{K / K_{0}} K^{*}$ which are positive at each $\mathfrak{p} \in \wp$. (Note that the elements of $\mathrm{N}_{K / K_{0}} K^{*}$ are positive at each $\mathfrak{p} \in \wp$.)

III. Two split unitary representations of odd type are equivalent if and only if they are equivalent as linear representations and their forms are isotypically equivalent.

Proof. Most of this follows easily from the fact that there is a bijection between Hermitian forms over $K$ and isotypic unitary representations, arising from $\underline{\hat{h}} \rightsquigarrow$ $\underline{\hat{h}} \otimes h_{0}^{t}$. The necessity of the condition in $\mathbf{I}$ that the indices be divisible by $n$ is a consequence of the fact that the $n \times n$ matrix $h_{0}$ is definite. Conversely, suppose that the indices of $h$ are $n r_{\mathfrak{p}}^{-}$and $n r_{\mathfrak{p}}^{+}$. If $n$ is odd, put

$$
\delta=\left(\frac{n}{g}\right)^{m}\left(\operatorname{det} h_{0}\right)^{m} \operatorname{det} h .
$$

Since $\operatorname{det} h_{0}$ is totally positive and $(\operatorname{det} h)(-1)^{n r_{\mathfrak{p}}^{-}}=1$ for all $\mathfrak{p} \in \wp$, we can apply the above lemma to find an Hermitian matrix $\underline{\hat{h}}$ with determinant $\delta$ and negative indices $r_{\mathfrak{p}}$. Since $\frac{n}{g}\left(\underline{\hat{h}} \otimes h_{0}\right)$ has the same determinant and indices as $h$, it is equivalent to $h$. Furthermore since the determinant and indices of $\underline{\hat{h}}$ are uniquely determined by those of $h$ (and $h_{0}$ ), we get II in the case of $n$ odd as well.

Suppose $n$ is even. The necessity of $\mathbf{I}$ is clear. Conversely, it is easy to see by Lemma 5.1 that there are Hermitian matrices $\underline{\hat{h}}$ with indices equal to those of $h$ divided by $n$, and then, for any such matrix, $\frac{n}{g}\left(\underline{\hat{h}} \otimes h_{0}\right)$ is equivalent to $h$. This proves I in the case of even $n$. Furthermore, any two such matrices $\underline{\hat{h}}$ have determinants differing by an element of $K_{0}$ which is positive at each $\mathfrak{p} \in \wp$. Conversely, if $\alpha_{0} \in K_{0}$ is positive at each $\mathfrak{p} \in \wp$ and $\underline{\hat{h}}$ is such an Hermitian matrix, then by the same lemma, there is another such matrix with determinant $\alpha_{0} \operatorname{det} \underline{\hat{h}}$. This proves II in the case of even $n$.

And III follows easily from II.

Theorem 5.3 (Split orthogonal representations). Suppose that $K$ is a global field and that the simple involution algebra component $A$ of $K G$ is $\cong \mathrm{M}(n, K)$. Let $V$ be an A-module of length $m$.

$\left(A,{ }^{-}\right)$symplectic.

I, II. Any invariant form on $V$ is hyperbolic, and the orthogonal representation it affords is unique up to equivalence.

$\left(A,{ }^{-}\right)$orthogonal.

I. Suppose that $h$ is a symmetric form on $V$.

If $n$ is odd, there is an invariant form $\cong h$ on $V$ if and only if

(i) $h \cong\left\langle\alpha h_{0}\right\rangle$ for some $\alpha \in K^{*}$ when $m=1$, and

(ii) $n \mid \operatorname{sgn}_{\mathfrak{p}} h$ for all real primes $\mathfrak{p}$ of $K$ when $m>1$.

If $n$ is even, there is an invariant form $\cong h$ on $V$ if and only if 
(iii) $\operatorname{det} h=\left(\operatorname{det} h_{0}\right)^{m}$, and

(iv) for all real primes $\mathfrak{p}$ of $K, n \mid r_{\mathfrak{p}}^{-}(h)$, and

(v) $\mathrm{S}_{\mathfrak{p}}(h)=\mathrm{S}_{\mathfrak{p}}\left(h_{0}\right)^{m}(-1,-1)_{\mathfrak{p}}^{\frac{n m(m-1)}{4}}$ for all $\mathfrak{p}$ at which $\operatorname{disc} h_{0}=$ 1.

II. If $h$ is invariant, there is (up to equivalence) only one orthogonal representation $G \rightarrow \mathbf{O}(V, h)$ if $n$ is odd, and there are an infinite number if $n$ is even.

III. Two split orthogonal representations of odd type are equivalent if and only if they are equivalent as linear representations and their underlying forms are isotypically equivalent.

Remark 5.4. 1. In (v), $\mathrm{S}_{\mathfrak{p}}\left(h_{0}\right)$ is well-defined, since for any $\alpha \in K^{*}, \mathrm{~S}_{\mathfrak{p}}\left(\alpha h_{0}\right)=$ $\mathrm{S}_{\mathfrak{p}}\left(h_{0}\right)$ when $n$ is even and disc $h_{0}=1$ at $\mathfrak{p}$.

2. In III, "odd type" can be weakened to " $A V=0$ for all simple orthogonal involution components $\left(A,^{-}\right)$of even degree."

Proof. If $\left(A,{ }^{-}\right)$is symplectic, $\underline{\hat{h}}$ must be skew symmetric and this case follows.

Suppose that $\left(A,{ }^{-}\right)$is orthogonal. If $n$ is odd, the necessity of (i) follows since $\underline{\hat{h}}=\langle\alpha\rangle$ implies $\underline{\hat{h}} \otimes h_{0}=\alpha h_{0}$. The necessity of (ii) is clear since $h \cong\left\langle\frac{n}{g}\left(\underline{\hat{h}} \otimes h_{0}\right)\right\rangle$ for some $\underline{\hat{h}}$ (and $h_{0}$ is positive definite).

The sufficiency of (i) is also clear. Suppose that $n$ is odd and $m>1$. By Lemmas 1.1 and 1.3 (iv), and the fact that $\operatorname{sgn}_{\mathfrak{p}}\left(\underline{\hat{h}} \otimes h_{0}\right)=n\left(\operatorname{sgn}_{\mathfrak{p}} \underline{\hat{h}}\right)$, the association $\underline{\hat{h}} \rightsquigarrow \underline{\hat{h}} \otimes h_{0}$ is injective (on equivalence classes of symmetric forms) since $n$ is odd. We now show that its image consists of all equivalence classes of symmetric forms on $V$ with signature at each real prime $\mathfrak{p}$ divisible by $n$. Suppose that $h$ is such a form, with signatures $n r_{\mathfrak{p}}$. We show the existence of a symmetric form $\underline{\hat{h}}$ such that $h \cong\left\langle\underline{\hat{h}} \otimes h_{0}\right\rangle$. Its determinant satisfies $\operatorname{det} h=(\operatorname{det} \underline{\hat{h}})^{n}\left(\operatorname{det} h_{0}\right)^{m}$, so $\operatorname{det} \underline{\hat{h}}=(\operatorname{det} h)\left(\operatorname{det} h_{0}\right)^{m}$. Similarly its Hasse-Witt invariant is determined by Lemma 1.3 (iv):

$$
\mathrm{S}(\underline{\hat{h}})=\mathrm{S}(h) \mathrm{S}\left(h_{0}\right)^{m}(\operatorname{det} \underline{\hat{h}},-1)_{K}^{n(n-1) / 2}\left(\operatorname{det} \underline{\hat{h}}, \operatorname{det} h_{0}\right)_{K}^{m n-1}\left(\operatorname{det} h_{0},-1\right)_{K}^{m(m-1) / 2},
$$

and its real signatures are $r_{\mathfrak{p}}$. We must check that the desired signatures are compatible with the determinant and Hasse-Witt invariant.

Let $r_{\mathfrak{p}}^{-}=\left(m-r_{\mathfrak{p}}\right) / 2$ be the negative index at $\mathfrak{p}$. At any real prime $\mathfrak{p}, \operatorname{det} h_{0}=1$, so

$$
\operatorname{det} \underline{\hat{h}}=\operatorname{det} h=(-1)^{n r_{\mathfrak{p}}^{-}}=(-1)^{r_{\mathfrak{p}}^{-}},
$$

as desired.

By (5.1) the Hasse invariant at $\mathfrak{p}$ is

$$
\begin{aligned}
\mathrm{S}_{\mathfrak{p}}(\underline{\hat{h}}) & =\mathrm{S}_{\mathfrak{p}}(h)(\operatorname{det} \underline{\hat{h}},-1)_{\mathfrak{p}}^{(n-1) / 2} \\
& =(-1)^{n r_{\mathfrak{p}}^{-}\left(n r_{\mathfrak{p}}^{-}-1\right) / 2}(-1)^{(n-1) r_{\mathfrak{p}}^{-} / 2} .
\end{aligned}
$$

It is easy to check that this is $=(-1)^{r_{\mathbf{p}}^{-}\left(r_{\mathbf{p}}^{-}-1\right) / 2}$, as desired.

The "local-global existence theorem" for quadratic forms ([19], Theorem 72:1) shows that $\underline{\hat{h}}$ exists. The upshot is that every form $h$ satisfying (i) or (ii) is equivalent to the $\operatorname{trace} \operatorname{trd}_{A / K} \hat{h}$ of an Hermitian form $\hat{h}$ over $\left(A,{ }^{-}\right)$and so is equivalent to an 
invariant form. The bijectivity implies II as well when $n$ is odd and $m>1$. And in the case $m=1$, II follows from the fact that $\left\langle\alpha h_{0}\right\rangle \cong\left\langle\beta h_{0}\right\rangle$ implies $\langle\alpha\rangle \cong\langle\beta\rangle$.

Now assume $n$ even. Suppose that $h$ is invariant. Since $h \cong\left\langle\frac{n}{g}\left(\underline{\hat{h}} \otimes h_{0}\right)\right\rangle$ for some $m \times m$ nonsingular symmetric matrix $\underline{\hat{h}}$, (iii) and (iv) follow at once. From Lemma 1.3 (i) and (iv),

$$
\begin{aligned}
\mathrm{S}(h) & =\mathrm{S}\left(\frac{n}{g}\left(\underline{\hat{h}} \otimes h_{0}\right)\right) \\
& =\mathrm{S}\left(h_{0}\right)^{m}\left(\operatorname{det} \underline{\hat{h}}, \operatorname{disc} h_{0}\right)\left(\operatorname{det} h_{0},-1\right)^{m(m-1) / 2}\left(\frac{n}{g}, \operatorname{disc} h_{0}\right)^{m} .
\end{aligned}
$$

If $\operatorname{disc} h_{0}=1$ at $\mathfrak{p}$, then $\operatorname{det} h_{0}=(-1)^{n / 2}$, and (v) follows at once.

Conversely, suppose that (iii), (iv) and (v) prevail. First we show there is $\delta \in K^{*}$ satisfying

(a) $\left(\delta, \operatorname{disc} h_{0}\right)=\mathrm{S}(h) \mathrm{S}\left(h_{0}\right)^{m}\left(\operatorname{det} h_{0},-1\right)^{m(m-1) / 2}\left(\frac{n}{g} \text {, disc } h_{0}\right)^{m}$, and

(b) the sign of $\delta$ at any real prime $\mathfrak{p}$ at which $\operatorname{disc} h_{0}=1$ is $(-1)^{r_{\mathfrak{p}}^{-}}$,

where $r_{\mathfrak{p}}^{-}(h)=n r_{\mathfrak{p}}^{-}$is the negative index of $h$. By Lemma 5.5 it is enough to find $\delta$ satisfying (a). If $\operatorname{disc} h_{0}$ is a square at $\mathfrak{p}$, the right side of (a) is 1 at $\mathfrak{p}$ by assumption (iv). By the Hilbert reciprocity law, and 19], 71:19 and 71:19a, there is a $\delta$ satisfying (a).

Now suppose $\mathfrak{p}$ is a real prime of $K$. Since $h_{0}$ is positive definite at all real primes,

$$
\mathrm{S}\left(h_{0}\right)=1, \quad \operatorname{det} h_{0}=1, \quad \operatorname{disc} h_{0}=(-1)^{n / 2} \quad \text { at } \mathfrak{p} .
$$

It follows from (a) that, also at $\mathfrak{p}$,

$$
\begin{aligned}
\left(\delta, \operatorname{disc} h_{0}\right) & =(\delta,-1)^{\frac{n}{2}} \\
& =\mathrm{S}(h)=(-1)^{\frac{n r_{\mathfrak{p}}^{-}}{2}}=(-1)^{r_{\mathfrak{p}}^{-}\left(\frac{n}{2}\right)} .
\end{aligned}
$$

Let $\hat{h}_{\mathfrak{p}}$ be a symmetric bilinear form of rank $m$ over $K_{\mathfrak{p}}$ with negative index $r_{\mathfrak{p}}^{-}$. If $\operatorname{disc} h_{0} \neq 1$ at $\mathfrak{p}$, then $n / 2$ must be odd, and it follows that $(\delta,-1)_{\mathfrak{p}}=(-1)^{r_{\mathfrak{p}}^{-}}=$ $\operatorname{det} \hat{h}_{\mathfrak{p}}$. Since each of $\delta$ and $\operatorname{det} \hat{h}_{\mathfrak{p}}$ can be considered to be \pm 1 at $\mathfrak{p}$, it follows that $\delta=\operatorname{det} \hat{h}_{\mathfrak{p}}$. On the other hand, if $\operatorname{disc} h_{0} i s$ a square at $\mathfrak{p}$, we get $\delta=\operatorname{det} \hat{h}_{\mathfrak{p}}$ at $\mathfrak{p}$ by (b).

It follows once more from the local-global existence theorem that there is a nonsingular symmetric matrix $\underline{\hat{h}}$ of rank $m$ over $K$ of determinant $\delta$ whose localizations at each real prime $\mathfrak{p}$ are the $\hat{h}_{\mathfrak{p}}$ just constructed. From (a), the expression for $\mathrm{S}\left(\frac{n}{g}\left(\underline{\hat{h}} \otimes h_{0}\right)\right)$ in $(\underline{5.2})$, the fact that $\operatorname{det} \frac{n}{g}\left(\underline{\hat{h}} \otimes h_{0}\right)=\left(\operatorname{det} h_{0}\right)^{m}$, and $r_{\mathfrak{p}}^{-}\left(\underline{\hat{h}} \otimes h_{0}\right)=n r_{\mathfrak{p}}^{-}(\underline{\hat{h}})=n r_{\mathfrak{p}}^{-}=r_{\mathfrak{p}}^{-}(h)$, it follows that $\operatorname{Tr}_{K G / K} \hat{h} \cong h$, where $\hat{h}$ is the Hermitian form over $\left(A,{ }^{-}\right)$on $K^{n \times m}$ with discriminant matrix $\underline{\hat{h}}$. Thus $\operatorname{Tr}_{K G / K} \hat{h}$ is an invariant form $\cong h$.

In fact there are an infinite number of such $\underline{\hat{h}}$ which are inequivalent, since we can choose the $S_{\mathfrak{p}}(\underline{\hat{h}})$ for $\mathfrak{p}$ finite arbitrarily (up to the Hilbert reciprocity law). Thus we get II for $n$ even: each invariant form $h$ supports an infinite number of inequivalent orthogonal representations.

III is a consequence of II, of course. 
Lemma 5.5. Let $\gamma \in K^{*}$ and let $(\delta, \gamma)$ be a fixed quaternion Brauer class over the algebraic number field $K$. Then the signs of $\delta$ at the real primes $\mathfrak{p}$ at which $\gamma$ is a square (i.e. for which $\gamma>_{\mathfrak{p}} 0$ ) can be arbitrarily prescribed.

Proof. Let "signs" $\epsilon_{\mathfrak{p}}= \pm 1$ be given for each real prime at which $\gamma$ is a square. For each such prime, choose $\alpha_{\mathfrak{p}}$ and $\beta_{\mathfrak{p}}$ in $K_{\mathfrak{p}}$ so that $\left(\alpha_{\mathfrak{p}}^{2}-\beta_{\mathfrak{p}}^{2} \gamma\right) \delta \epsilon_{\mathfrak{p}}>_{\mathfrak{p}} 0$. Apply the weak approximation theorem to find $\alpha$ and $\beta$ in $K$ such that $\left(\alpha^{2}-\beta^{2} \gamma\right) \delta \epsilon_{\mathfrak{p}}>_{\mathfrak{p}} 0$ for each $\mathfrak{p}$, and set $\delta^{\prime}=\left(\alpha^{2}-\beta^{2} \gamma\right) \delta$. Then

$$
\left(\delta^{\prime}, \gamma\right)=\left(\alpha^{2}-\beta^{2} \gamma, \gamma\right)(\delta, \gamma)=(\delta, \gamma),
$$

since, if $\gamma$ is not a square in $K, \alpha^{2}-\beta^{2} \gamma$ is a norm in $K(\sqrt{\gamma}) / K$. Furthermore,

$$
\delta^{\prime} \epsilon_{\mathfrak{p}}=\left(\alpha^{2}-\beta^{2} \gamma\right) \delta \epsilon_{\mathfrak{p}}>_{\mathfrak{p}} 0
$$

for any real $\mathfrak{p}$ at which $\gamma$ is a square.

The Hasse Principle. We now prove the Hasse Principle for equivariant representations.

The completion of the fixed field $K_{0}$ of the involution at any discrete or Archimedean prime $\mathfrak{p}$ is denoted by $K_{0 \mathfrak{p}}$. Similarly $V_{\mathfrak{p}}=K_{0 \mathfrak{p}} \otimes_{K_{0}} V, A_{\mathfrak{p}}=K_{0 \mathfrak{p}} \otimes_{K_{0}} A, \ldots$ - these are all modules over $K_{\mathfrak{p}}=K_{0 \mathfrak{p}} \otimes_{K_{0}} K$. The latter ring has a $K$-involution given by $\overline{\alpha \otimes \beta}=\alpha \otimes \bar{\beta}$. If $\mathfrak{p}$ splits in $K$ - which implies that the involution on $K$ is not the identity $-K_{\mathfrak{p}}$ is the hyperbolic involution algebra $K_{0 \mathfrak{p}} \oplus K_{0 \mathfrak{p}}$. Otherwise it is the completion of $K$ with respect to the unique extension of $\mathfrak{p}$ to $K$. Similarly,

$$
(K G)_{\mathfrak{p}}=K_{0 \mathfrak{p}} \otimes_{K_{0}} K G=K_{\mathfrak{p}} \otimes_{K} K G=K_{\mathfrak{p}} G
$$

is also a hyperbolic involution algebra if $\mathfrak{p}$ splits in $K$. In this case any Hermitian form over $K_{\mathfrak{p}} G$ is hyperbolic, and so is determined by the isomorphism class of the $K_{\mathfrak{p}} G$-module $V_{\mathfrak{p}}$ ([14, 2.3), and so by the isomorphism class of the $K G$-module $V$ itself.

Of course if the involution on $K$ is the identity, then $K_{0}=K$ and $K_{0 \mathfrak{p}}=K_{\mathfrak{p}}$.

Note that any sesquilinear form $f: V \times V \rightarrow A$ over the $K$-involution algebra $A$ has a unique extension to a sesquilinear form

$$
f_{\mathfrak{p}}: V_{\mathfrak{p}} \times V_{\mathfrak{p}} \rightarrow A_{\mathfrak{p}}
$$

determined by

$$
f_{\mathfrak{p}}(\alpha \otimes v, \beta \otimes u)=\alpha \beta \otimes f(v, u),
$$

where $\alpha, \beta \in K_{0 \mathfrak{p}}$.

We can also extend an equivariant representation $\rho: G \rightarrow \mathbf{I}(V, h)(\mathbf{I}=\mathbf{O}, \mathbf{S p}$, or $\mathbf{U})$ to an equivariant representation $\rho_{\mathfrak{p}}: G \rightarrow \mathbf{I}\left(V_{\mathfrak{p}}, h_{\mathfrak{p}}\right)$ in the obvious way,

$$
\rho_{\mathfrak{p}}(s)(\alpha \otimes v)=\alpha \otimes \rho(s) v .
$$

It is clear that $\rho_{\mathfrak{p}}(s) \in \mathbf{I}\left(V_{\mathfrak{p}}, h_{\mathfrak{p}}\right)$ and that $\rho_{\mathfrak{p}}$ is a homomorphism.

Theorem 5.6 (Hasse Principle for equivariant representations). Let $G$ be an arbitrary finite group and $K$ a global field. Let $\rho: G \rightarrow \mathbf{I}(V, h)$ be an equivariant representation, and $\mathrm{P}$ the set of all equivariant representations on $V$ which are linearly equivalent to $\rho$. Then the "Hasse Principle"

$$
\text { for all } \rho^{\prime} \in \mathrm{P}, \quad \rho \cong \rho^{\prime} \Leftrightarrow \rho_{\mathfrak{p}} \cong \rho_{\mathfrak{p}}^{\prime} \text { for all primes } \mathfrak{p} \text { of } K_{0}
$$

holds if and only if $A V=0$ for every simple direct summand $A$ of $K G$ which satisfies the following: 
$\left(^{*}\right) A$ is a matrix algebra over a quaternion algebra which is nonsplit at more than 2 primes (of its center), and the restriction of the canonical involution on $K G$ to $A$ is an involution of orthogonal type if $\rho$ is orthogonal, and of symplectic type if $\rho$ is symplectic.

Remark 5.7. In particular, this means that the Hasse Principle always holds for unitary representations.

Proof. Suppose that

$$
\rho: G \rightarrow \mathbf{I}(V, h), \quad \rho^{\prime}: G \rightarrow \mathbf{I}\left(V^{\prime}, h^{\prime}\right)
$$

are two equivariant representations which are "of the same type" ( $h$ and $h^{\prime}$ both symmetric, or both Hermitian, ...) and that they are linearly equivalent. In fact we assume that they are equal (as linear representations). Let $\epsilon=1$ if $h$ and $h^{\prime}$ are symmetric or Hermitian, -1 if they are skew symmetric or skew Hermitian. We shall refer to $h$ and $h^{\prime}$ as $\epsilon$-Hermitian in both cases.

According to $\$ 1.3$ there are simple summands $A_{1}, \ldots, A_{k}$ of $K G$ stable under - and forms $\breve{h}_{1}, \ldots, \breve{h}_{k}$ over the division algebras $D_{1}, \ldots, D_{k}$, with $D_{i}$ Brauer equivalent to $A_{i}$, whose (ordinary) equivalence classes characterize the equivariant equivalence classes of $\rho$. We now look more closely at the last step in this association, the Hermitian Morita equivalence of $\hat{h}_{i}$ and $\breve{h}_{i}$, using the "Hermitian Morita context" of Lemma 1.8

There is an $\epsilon_{i}$-Hermitian matrix $\underline{h}_{0 i} \in A_{i}=\mathrm{M}\left(n_{i}, D_{i}\right)$ such that $\bar{a}=\underline{h}_{0 i}^{-1} a^{*} \underline{h}_{0 i}$ for all $a \in A_{i}$. Let $V_{0 i}$ be the simple $A_{i}$-module $D_{i}^{n_{i} \times 1}$. It is an $\left(A_{i}, D_{i}\right)$-bimodule. By Lemma 1.8, the nonsingular $\epsilon_{i}$-Hermitian form $h_{0 i}: V_{0 i} \times V_{0 i} \rightarrow A_{i}$ defined by $h_{0 i}(u, v)=u v^{*} \underline{h}_{0 i}$ is $\epsilon_{i}$-Hermitian and, if $D_{i}$ is a quaternion algebra and the involution on $A_{i}$ is of the first kind, the adjoint involution ${ }^{-}$on $D_{i}$ is conjugation; in this latter case we note that $\epsilon_{i}=1$ respectively -1 if $\left(A,{ }^{-}\right)$is symplectic respectively orthogonal. Twist the action of $A_{i}$ and $D_{i}$ on $V_{0 i}$ using their involutions, and let $\bar{V}_{0 i}$ denote the resulting $\left(D_{i}, A_{i}\right)$-bimodule.

By the Hermitian Morita theory, there is a nonsingular $\epsilon_{i}$-Hermitian form $H_{0 i}$ : $\bar{V}_{0 i} \times \bar{V}_{0 i} \rightarrow D_{i}$ whose adjoint involution on $A_{i}$ is ${ }^{-}$and which satisfies the Hermitian Morita associative relationship

$$
h_{0 i}(u, v) w=u H_{0 i}(\bar{v}, \bar{w}) \quad \text { for all } u, v, w \in V_{0 i} .
$$

It effects an equivalence between the category ${ }_{A_{i}} \mathcal{H}_{\epsilon}$ of $\epsilon$-Hermitian forms over $A_{i}$ and the category $D_{i} \mathcal{H}_{\epsilon \epsilon_{i}}$ of $\epsilon \epsilon_{i}$-Hermitian forms over $D_{i}$, via the map $\hat{g} \rightsquigarrow H_{0 i} \hat{g}$ (product of forms-cf. [8], §2). This is the equivalence described in Lemma 1.8]

By Table 1 in $\$ 1.1$ and 10.4.6, [21], the Hasse Principle applies to the forms $H_{0 i} \hat{g}$ unless they are skew Hermitian $\left(\epsilon \epsilon_{i}=-1\right)$ and $D_{i}$ is a quaternion division algebra which is nonsplit at more than 2 primes. These exceptional cases are precisely those arising from $\left(^{*}\right)$, and so, in order to prove the sufficiency of the condition in the theorem, we assume that $A_{i} V=0$ for any such exceptional $i, 1 \leq i \leq k$. Of course this means also that $\hat{h}_{i}=0$.

It follows that $\rho \cong \rho^{\prime}$ if and only if $\left(H_{0 i} \hat{h}_{i}\right)_{\mathfrak{P}} \cong\left(H_{0 i} \hat{h}_{i}^{\prime}\right)_{\mathfrak{P}}$ for $1 \leq i \leq k$ and all primes $\mathfrak{P}$ of $L_{i 0}$, where $L_{i}$ is the center of $D_{i}$ and $L_{i 0}$ is the subfield of elements fixed by the involution of $L_{i}$. We now wish to show that this is equivalent with

$$
\rho \cong \rho^{\prime} \Leftrightarrow\left(H_{0 i} \hat{h}_{i}\right)_{\mathfrak{p}} \cong\left(H_{0 i} \hat{h}_{i}^{\prime}\right)_{\mathfrak{p}} \text { for } 1 \leq i \leq k \text { and all primes } \mathfrak{p} \text { of } K_{0} .
$$

In doing this we shall drop the index $i$ from $L_{i}, L_{i 0}, D_{i}$, etc., in order to simplify the notation. 
By [2], p. 57, there is an isomorphism $K_{0 \mathfrak{p}} \otimes_{K_{0}} L_{0} \cong \bigoplus_{\mathfrak{P} \mid \mathfrak{p}} L_{0 \mathfrak{P}}$, which implies an isomorphism

$$
L_{\mathfrak{p}} \cong \bigoplus_{\mathfrak{P} \mid \mathfrak{p}} L_{\mathfrak{P}} \quad\left(L_{\mathfrak{p}}=K_{0 \mathfrak{p}} \otimes_{K_{0}} L, \quad L_{\mathfrak{P}}=L_{0 \mathfrak{P}} \otimes_{L_{0}} L\right)
$$

given by

$$
\alpha_{\mathfrak{p}} \otimes \beta \mapsto\left(\iota_{1} \alpha_{\mathfrak{p}} \otimes \beta, \ldots, \iota_{q} \alpha_{\mathfrak{p}} \otimes \beta\right),
$$

where $\iota_{j}: K_{0 \mathfrak{p}} \rightarrow L_{0 \mathfrak{P}_{j}}$ is the inclusion. We identify $L_{\mathfrak{p}}$ and $\bigoplus_{\mathfrak{P} \mid \mathfrak{p}} L_{\mathfrak{P}}$ via this isomorphism. Similarly we identify

$$
D_{\mathfrak{p}}=\bigoplus_{\mathfrak{P} \mid \mathfrak{p}} D_{\mathfrak{P}} \quad \text { and } \quad W_{\mathfrak{p}}=\bigoplus_{\mathfrak{P} \mid \mathfrak{p}} W_{\mathfrak{P}},
$$

where $W$ is a vector space over $D$, via the isomorphisms

$$
\alpha_{\mathfrak{p}} \otimes d \mapsto\left(\iota_{1} \alpha_{\mathfrak{p}} \otimes d, \ldots, \iota_{q} \alpha_{\mathfrak{p}} \otimes d\right) \quad \text { and } \quad \alpha_{\mathfrak{p}} \otimes w \mapsto\left(\iota_{1} \alpha_{\mathfrak{p}} \otimes w, \ldots, \iota_{q} \alpha_{\mathfrak{p}} \otimes w\right)
$$

These identifications are compatible with the inclusion of $D$ respectively $W$ on both sides, as well as with the action of $D_{\mathfrak{p}}$ on $W_{\mathfrak{p}}$ and $\bigoplus_{\mathfrak{P} \mid \mathfrak{p}} D_{\mathfrak{P}}$ on $\bigoplus_{\mathfrak{P} \mid \mathfrak{p}} W_{\mathfrak{P}}$.

Let $f: W \times W \rightarrow D$ be a sesquilinear form.

Lemma 5.8. (a) The sesquilinear module $\left(W_{\mathfrak{p}}, f_{\mathfrak{p}}\right)$ is the orthogonal direct sum of the sesquilinear modules $\left(W_{\mathfrak{P}}, f_{\mathfrak{P}}\right)$. Briefly, $f_{\mathfrak{p}}=\perp_{\mathfrak{P} \mid \mathfrak{p}} f_{\mathfrak{P}}$.

(b) Let $g: U \times U \rightarrow D$ be another sesquilinear form. Then $f_{\mathfrak{p}} \cong g_{\mathfrak{p}}$ if and only if $f_{\mathfrak{P}} \cong g_{\mathfrak{P}}$ for all primes $\mathfrak{P} \mid \mathfrak{p}$ of $L_{0}$.

Proof. First of all, $f_{\mathfrak{P}}: W_{\mathfrak{P}} \times W_{\mathfrak{P}} \rightarrow D_{\mathfrak{P}} \subset D_{\mathfrak{p}}$ and so can be considered as a sesquilinear form over $D_{\mathfrak{p}}$. Then

$$
\begin{aligned}
\left(\perp_{\mathfrak{P} \mid \mathfrak{p}}\right. & \left.f_{\mathfrak{P}}\right)\left(\alpha \otimes w, \alpha^{\prime} \otimes w^{\prime}\right) \\
= & \left(f_{\mathfrak{P}_{1}}\left(\iota_{1} \alpha \otimes w, \iota_{1} \alpha^{\prime} \otimes w^{\prime}\right), \ldots, f_{\mathfrak{P}_{q}}\left(\iota_{q} \alpha \otimes w, \iota_{q} \alpha^{\prime} \otimes w^{\prime}\right)\right) \\
= & \left(\iota_{1} \alpha \alpha^{\prime} \otimes f\left(w, w^{\prime}\right), \ldots, \iota_{q} \alpha \alpha^{\prime} \otimes f\left(w, w^{\prime}\right)\right) \\
= & \alpha \alpha^{\prime} \otimes f\left(w, w^{\prime}\right)=f_{\mathfrak{p}}\left(\alpha \otimes w, \alpha^{\prime} \otimes w^{\prime}\right)
\end{aligned}
$$

which proves (a). And (b) follows, since $W_{\mathfrak{P}}=D_{\mathfrak{P}} W_{\mathfrak{p}}$ and $U_{\mathfrak{P}}=D_{\mathfrak{P}} U_{\mathfrak{p}}$.

It follows then that (5.4) holds.

Lemma 5.9. Suppose that $A$ and $B$ are $K$-involution algebras, that $f: U \times U \rightarrow B$ is a sesquilinear form admitting $A$, and that $g: V \times V \rightarrow A$ is a sesquilinear form. If $\mathfrak{p}$ is a prime of $K_{0}$, the forms $(f g)_{\mathfrak{p}}$ and $f_{\mathfrak{p}} g_{\mathfrak{p}}$ over $B_{\mathfrak{p}}$ are canonically equivalent.

Proof. There is a standard $K_{0 \mathfrak{p}}$-isomorphism $\phi:\left(U \otimes_{A} V\right)_{\mathfrak{p}} \rightarrow U_{\mathfrak{p}} \otimes_{A_{\mathfrak{p}}} V_{\mathfrak{p}}$ taking $\alpha \otimes(u \otimes v)$ to $(\alpha \otimes u) \otimes(1 \otimes v) \quad\left(\alpha \in K_{0 \mathfrak{p}}, u \in U, v \in V\right)$. It is easy to check that $\phi$ is an isomorphism of $B_{\mathfrak{p}}$-modules, and that it is also an equivalence between the forms $(f g)_{\mathfrak{p}}$ and $f_{\mathfrak{p}} g_{\mathfrak{p}}$. (Recall that the definition of the product $f g:\left(U \otimes_{A} V\right) \times$ $\left(U \otimes_{A} V\right) \rightarrow B$ is $\left.(f g)\left(u \otimes v, u^{\prime} \otimes v^{\prime}\right)=f\left(u g\left(v, v^{\prime}\right), u^{\prime}\right)\right)$.

We now prove that the Hasse Principle holds, under the assumption that $A V=0$ for every simple direct summand $A$ of $K G$ which satisfies $(*)$. Any such summand is certainly one of the $A_{i}, 1 \leq i \leq k$, defined in 1.3 , let $A_{i}, 1 \leq i \leq m$, be the remaining $A_{i}$. From (5.4) and Lemma 5.9, we get

$$
\rho \cong \rho^{\prime} \Leftrightarrow H_{0 i \mathfrak{p}} \hat{h}_{i \mathfrak{p}} \cong H_{0 i \mathfrak{p}} \hat{h}_{i \mathfrak{p}}^{\prime} \text { for all } \mathfrak{p} \text { and } 1 \leq i \leq m \text {. }
$$


Now consider $\rho_{\mathfrak{p}}: G \rightarrow \mathbf{I}\left(V_{\mathfrak{p}}, h_{\mathfrak{p}}\right), h_{\mathfrak{p}}: V_{\mathfrak{p}} \times V_{\mathfrak{p}} \rightarrow K_{\mathfrak{p}}$. Then $\widehat{h_{\mathfrak{p}}}: V_{\mathfrak{p}} \times V_{\mathfrak{p}} \rightarrow$ $K_{\mathfrak{p}} G=\bigoplus_{i=1}^{t} A_{i \mathfrak{p}}$ and $\left(\widehat{h_{\mathfrak{p}}}\right)_{i}: A_{i \mathfrak{p}} V_{\mathfrak{p}} \times A_{i \mathfrak{p}} V_{\mathfrak{p}} \rightarrow A_{i \mathfrak{p}}$. Furthermore, it is easy to check that $\rho_{\mathfrak{p}} \cong \rho_{\mathfrak{p}}^{\prime}$ if and only if $\left(\widehat{h_{\mathfrak{p}}}\right)_{i} \cong\left(\widehat{h_{\mathfrak{p}}^{\prime}}\right)_{i}$ for all $i, 1 \leq i \leq t$.

Now suppose that $i>k$ (cf. $\$ 1.3$ ). If $A_{i}$ is a hyperbolic involution algebra, so is $A_{i \mathrm{p}}$. Otherwise $A_{i}$ is of index 1 with a symplectic respectively orthogonal involution (which implies that the involution on $K$ is the identity), and so $A_{i \mathfrak{p}}$ is likewise of index 1 with a symplectic respectively orthogonal involution, or is a direct sum of such involution algebras. Thus if $\rho$ is orthogonal respectively symplectic, the forms $\left(\widehat{h_{\mathfrak{p}}}\right)_{i}$ and $\left(\widehat{h_{\mathfrak{p}}^{\prime}}\right)_{i}$ arising from these algebras are determined by the $K_{\mathfrak{p}} G$-module structure of the spaces on which they are defined (cf. \$1.3).

Thus $\rho_{\mathfrak{p}} \cong \rho_{\mathfrak{p}}^{\prime}$ if and only if $\left(\widehat{h_{\mathfrak{p}}}\right)_{i} \cong\left(\widehat{h_{\mathfrak{p}}^{\prime}}\right)_{i}$ for all $i, 1 \leq i \leq k$.

The next step is to show that $\left(\widehat{h_{\mathfrak{p}}}\right)_{i}=\hat{h}_{i \mathfrak{p}}$. Now $\hat{h}_{i \mathfrak{p}}$ is defined on $\left(A_{i} V\right)_{\mathfrak{p}}=$ $K_{0 \mathfrak{p}} \otimes A_{i} V$, which we identify with a submodule of $K_{0 \mathfrak{p}} \otimes V=V_{\mathfrak{p}}$ in the usual way. Note that $A_{i \mathfrak{p}} V_{\mathfrak{p}}$ is spanned, as an Abelian group, by elements of the form $\left(\alpha \otimes a_{i}\right)(\beta \otimes v)=\alpha \beta \otimes a_{i} v \in\left(A_{i} V\right)_{\mathfrak{p}}$. It follows that $A_{i \mathfrak{p}} V_{\mathfrak{p}}=\left(A_{i} V\right)_{\mathfrak{p}}$. We can now show that $\left(\widehat{h_{\mathfrak{p}}}\right)_{i}=\hat{h}_{i \mathfrak{p}}$. Let $\alpha, \beta \in K_{0 \mathfrak{p}}$ and $u, v \in A_{i} V$. Then (cf. (1.2))

$$
\begin{aligned}
\widehat{h_{\mathfrak{p}}}(\alpha & \otimes u, \beta \otimes v) \\
& =\sum_{s} h_{\mathfrak{p}}\left(\rho_{\mathfrak{p}}\left(s^{-1}\right)(\alpha \otimes u), \beta \otimes v\right) s \\
& =\sum_{s} h_{\mathfrak{p}}\left(\alpha \otimes \rho\left(s^{-1}\right) u, \beta \otimes v\right) s \\
& =\sum_{s} \alpha \beta \otimes h\left(\rho\left(s^{-1}\right) u, v\right) s=\alpha \beta \otimes \sum_{s} h\left(\rho\left(s^{-1}\right) u, v\right) s \\
& =\alpha \beta \otimes \hat{h}(u, v)=\hat{h}_{i \mathfrak{p}}(\alpha \otimes u, \beta \otimes v) .
\end{aligned}
$$

Thus

$$
\rho_{\mathfrak{p}} \cong \rho_{\mathfrak{p}}^{\prime} \quad \Leftrightarrow \quad \hat{h}_{i \mathfrak{p}} \cong \hat{h}_{i \mathfrak{p}}^{\prime} \text { for all } i, 1 \leq i \leq k
$$

The forms $h_{0 i}: V_{0 i} \times V_{0 i} \rightarrow A_{i}$ and $H_{0 i}: \bar{V}_{0 i} \times \bar{V}_{0 i} \rightarrow D_{i}$ are nonsingular $\epsilon_{i^{-}}$ Hermitian forms which satisfy the associativity relationship (5.3). The forms $h_{0 i \mathfrak{p}}$ and $H_{0 i p}$ also satisfy the associativity relationship:

$$
\begin{aligned}
h_{0 i \mathfrak{p}}(\alpha \otimes u, \beta \otimes v)(\gamma \otimes w) & =\left(\alpha \beta \otimes h_{0 i}(u, v)\right)(\gamma \otimes w)=\alpha \beta \gamma \otimes h_{0 i}(u, v) w \\
& =\alpha \beta \gamma \otimes\left(u H_{0 i}(\bar{v}, \bar{w})\right)=(\alpha \otimes u)\left(\beta \gamma \otimes H_{0 i}(\bar{v}, \bar{w})\right) \\
& =(\alpha \otimes u) H_{0 i \mathfrak{p}}(\beta \otimes \bar{v}, \gamma \otimes \bar{w}) .
\end{aligned}
$$

It follows from the Hermitian Morita theory that $h_{0 i \mathfrak{p}}$ and $H_{0 i \mathfrak{p}}$ induce a category equivalence between the category of $\epsilon$-Hermitian forms over $A_{i \mathfrak{p}}$ and the category of $\epsilon_{i} \epsilon$-Hermitian forms over $D_{i \mathfrak{p}}$. This implies that

$$
\rho_{\mathfrak{p}} \cong \rho_{\mathfrak{p}}^{\prime} \Leftrightarrow H_{0 i \mathfrak{p}} \hat{h}_{i \mathfrak{p}} \cong H_{0 i \mathfrak{p}} \hat{h}_{i \mathfrak{p}}^{\prime} \text { for all } i, 1 \leq i \leq k .
$$

This together with (5.5) shows that $\rho \cong \rho^{\prime}$ if and only if $\rho_{\mathfrak{p}} \cong \rho_{\mathfrak{p}}^{\prime}$ for all $\mathfrak{p}$ (since $\hat{h}_{i}=0$ for $\left.m<i \leq k\right)$.

Conversely, suppose that $A_{i} V \neq 0$ for some $A_{i}$ satisfying (*). In particular, $\rho$ is orthogonal $\left(\epsilon=1, \epsilon_{i}=-1\right)$ or symplectic $\left(\epsilon=-1, \epsilon_{i}=1\right)$. Then $H_{0 i} \hat{h}_{i}$ is a skew Hermitian form over $D_{i}$ on $\bar{V}_{0 i} \otimes A_{i} V$, and there is another nonsingular skew Hermitian form over $D_{i}$ on the same space, which is locally equivalent to $H_{0 i} \hat{h}_{i}$ 
at all primes $\mathfrak{P}$ but not globally equivalent to it (cf. [21], 10.4.6). By Morita equivalence there is an $\epsilon$-Hermitian form $\hat{h}_{i}^{\prime \prime}$ over $A_{i}$ on $A_{i} V$ which is locally but not globally equivalent to $\hat{h}_{i}$. This gives rise in an obvious way to an orthogonal or symplectic representation $\rho^{\prime \prime}: G \rightarrow \mathbf{U}\left(V, h^{\prime \prime}\right)$ which is locally equivalent to $\rho$ at all $\mathfrak{p}$ but not globally equivalent. Namely, one defines $\hat{h}^{\prime \prime}=\perp_{j} \hat{h}_{j}^{\prime \prime}$, where $\hat{h}_{j}^{\prime \prime}=\hat{h}_{j}$ for all $j \neq i$, and then $h^{\prime \prime}=\operatorname{Tr}_{K G / K} \circ \hat{h}^{\prime}(\mathrm{cf}$. (1.3) ). This finishes the proof of Theorem 5.6 .

Corollary 5.10. The Hasse Principle always holds in the following cases:

(a) The representations are unitary.

(b) The characteristic of $K$ is $\neq 0$.

(c) The representations are of odd type.

(d) $G$ is nilpotent and $K$ contains either a cubic or quartic root of unity.

Proof. If the representation is unitary, $(*)$ does not apply. If the characteristic is $\neq 0$, the $A_{i}$ all have index 1 and so again $\left(^{*}\right)$ does not apply. Suppose that the characteristic is 0 . If the degree of every absolutely irreducible character is odd, no $A_{i}$ is similar to a quaternion algebra. In (d) the $A_{i}$ also have index $1-$ see [7], 14.5, p. 77 .

\section{EquiVARIANT REPRESENTATIONS OF THE SYMMETRIC GROUP.}

As with linear and projective representations, the equivariant representation theory of $\mathrm{S}_{r}$ is especially explicit - the essential points are that all representations are split and that one can calculate the invariant symmetric form $h_{0}$ explicitly for an irreducible representation $\rho: \mathrm{S}_{r} \rightarrow \mathbf{G L}(V)$. This is of course the same matrix involved in the involution $\bar{a}=h_{0}^{-1} a^{*} h_{0}$ on the direct summand of $K G$ corresponding to $\rho$, induced by the canonical involution of $K G$. The procedure for the calculation of $h_{0}$ is described in [10]; we illustrate it by an example. This method applies if char $K=0$ or $p>r$.

We note that when $G=\mathbf{S}_{r}$, every simple involution algebra component $A$ of $K G$ is orthogonal. In characteristic 0 , this follows from the existence of a nonsingular invariant symmetric form on a simple $A$-module - in fact it can be assumed to be a positive definite form defined over $\mathbb{Q}$ - and from the fact that such a form is unique up to scalar multiples since $A$ is split. In characteristic $p$, there is also a nonsingular invariant symmetric form on any simple module, which is described explicitly later in this section via the example.

If $K$ has a nontrivial involution and $K \mathbf{S}_{r}$ has the corresponding canonical involution, then every simple involution component $A$ of $K \mathbf{S}_{r}$ is again a simple algebra. This follows from the fact that $K \mathbf{S}_{r}$ is split over the prime subfield, so $A=K \otimes_{K_{0}} A_{0}$, where $A_{0}$ is a simple involution component of $K_{0} \mathbf{S}_{r}$, and the involution on $A$ is the tensor product of the involutions on $K$ and $A_{0}$. This implies that the Hermitian matrix $h_{0}$ for the unitary involution on $A$ is identical with the symmetric matrix for the involution of the first kind on $A_{0}$.

All of the facts cited in the following about representations of $\mathrm{S}_{r}$ can be found in [10].

We recall that each irreducible representation of $\mathrm{S}_{r}$ arises from a partition $\alpha=$ $\left[\alpha_{1}, \alpha_{2}, \ldots, \alpha_{k}\right]$ of $r$; thus the $\alpha_{i}$ are positive integers satisfying $\alpha_{1} \geq \alpha_{2} \geq \cdots \geq \alpha_{k}$ and $\sum_{i} \alpha_{i}=r$ (cf. [10], pp. 350 and 376). We shall describe the calculation of $h_{0}$ 
through an example, namely the partition

$$
\alpha=[3,2,2]
$$

of 7 , which gives rise to an irreducible representation of $\mathrm{S}_{7}$. The standard $\alpha$-tableaus are the arrays

\begin{tabular}{|c|c|c|c|c|c|c|c|c|c|c|c|c|c|}
\hline 12 & 3 & 1 & 2 & 3 & 1 & 2 & 4 & & 1 & 2 & 5 & 1 & 2 \\
\hline 5 & & 4 & 6 & & 3 & 5 & & & 3 & 4 & & 3 & 6 \\
\hline $\begin{array}{ll}6 & 7\end{array}$ & & 5 & 7 & & 6 & 7 & & & 6 & 7 & & 4 & 7 \\
\hline 2 & 6 & 1 & 2 & 6 & 1 & 2 & 7 & & 1 & 3 & 4 & 1 & 3 \\
\hline 4 & & 3 & 5 & & 3 & 4 & & & 2 & 5 & & 2 & 6 \\
\hline 7 & & 4 & 7 & & 5 & 6 & & & 6 & 7 & & 5 & 7 \\
\hline 3 & 5 & 1 & 3 & 5 & 1 & 3 & 6 & & 1 & 3 & 7 & 1 & 3 \\
\hline 4 & & 2 & 6 & & 2 & 4 & & & 2 & 4 & & 2 & 5 \\
\hline 7 & & 4 & 7 & & 5 & 7 & & & 5 & 6 & & 4 & 6 \\
\hline & & & 1 & 4 & 5 & 1 & 4 & 6 & 7 & & & & \\
\hline & & & 2 & 6 & & 2 & 5 & & & & & & \\
\hline & & & 3 & 7 & & 3 & 7 & & & & & & \\
\hline
\end{tabular}

They are characterized by the fact that the row lengths constitute the given partition of 7 , and the integers in each row and in each column are increasing. The number of standard $\alpha$-tableaus, 21 , is the degree of the corresponding representation ([10], 3.1.13, p. 107).

There is an orthogonal basis $u_{1}, \cdots, u_{21}$ (with respect to $h_{0}$ ) of the representation space for which one can calculate $h_{0}\left(u_{i}, u_{i}\right)$. One starts with the $i^{\text {th }}$ standard tableau; then

$$
h_{0}\left(u_{i}, u_{i}\right)=\prod_{x=1}^{7} \prod_{k} \frac{h(x, k)}{h(x, k)-1},
$$

where $h(x, k)$ is a "hook length" defined as follows. $k=1,2,3$ is the number of a row. $h(x, k)$ is not defined (and hence does not appear in the above product) unless the integer $x$ is in a row lower than row $k$; thus $h(1, k)$ and $h(x, 3)$ are not defined for any $k$ and $x$.

Suppose $x$ is in a row lower than row $k$. Then $h(x, k)$ is the number of integers in the "hook" consisting of

(i) the integer $x$,

(ii) the integers above $x$ up to row $k$ (but not in rows $1, \ldots, k-1$ ) in the same column as $x$, and

(iii) the integers $\leq x$ in row $k$ to the right of the column of $x$.

For example, in the $8^{\text {th }}$ standard tableau in the above display

$\begin{array}{lll}1 & 2 & 6 \\ 3 & 5 & \\ 4 & 7 & \end{array}$


we have $h(4,1)=4, h(4,2)=2$ (and of course $h(4,3)$ is not defined). It is easy to check that

$$
h_{0}\left(u_{8}, u_{8}\right)=\prod_{x=2}^{7} \prod_{k=1}^{2} \frac{h(x, k)}{h(x, k)-1}=\left(\frac{3}{2}\right)\left(\frac{4}{3} \cdot \frac{2}{1}\right)\left(\frac{2}{1}\right)\left(\frac{4}{3} \cdot \frac{2}{1}\right)=\frac{2^{6}}{3}
$$

where the parenthesized factors correspond respectively to $x=3,4,5,7$.

In this way one gets for $h_{0}\left(u_{i}, u_{i}\right), 1 \leq i \leq 21$,

$$
\begin{array}{r}
10, \quad 40 / 3, \quad 45 / 4, \quad 15, \quad 15, \quad 16, \\
16, \quad 64 / 3, \quad 18, \quad 24, \quad 15, \quad 20, \\
20, \quad 64 / 3, \quad 64 / 3, \quad 256 / 9, \quad 24, \quad 32,
\end{array}
$$

$24, \quad 32, \quad 36$.

After adjusting by squares,

$$
h_{0}=\langle 10,30,5,15,15,1,1,3,2,6,15,5,5,3,3,1,6,2,6,2,1\rangle .
$$

Thus one can compute the determinant and Hasse symbol of $h_{0}$; they are 1 and $(3,3)_{K}$ respectively. This means that, over a local or global field

$$
h_{0} \cong\langle 1,1, \ldots, 1,3,3\rangle \text {. }
$$

\section{REFERENCES}

[1] N. Bourbaki, Algèbre (modules et anneaux semi-simples), Actualités Scientifiques et Industrielles, no. 1261, Hermann, 1958. MR 20:4576]

[2] J. W. S. Cassels and A. Fröhlich, Algebraic number theory, Conference Proceedings, Academic Press Inc., London, 1967. MR 35:6500

[3] Charles W. Curtis and Irving Reiner, Representation theory of finite groups and associative algebras, Pure and Applied Mathematics, vol. XI, Interscience Publishers, John Wiley and Sons, New York, 1962. MR 26:2519

[4] Andreas W. M. Dress, Induction and structure theorems for orthogonal representations of finite groups, Annals of Mathematics 102 (1975), 291-325. MR 52:8235

[5] Martin Epkenhans, Spurformen über Lokalen Körpern, Schriftenreihe der Mathematischen Instituts der Universität Münster, vol. 44, Math. Inst. Univ. Münster, 1987. MR 88j:11017

[6] - Trace forms of normal extensions over local fields, Linear and Multilinear Algebra 24 (1989), 103-116. MR 90m:11188

[7] Walter Feit, Characters of Finite Groups, Mathematics Lecture Notes, W.A.Benjamin Inc., New York, 1967. MR 36:2715

[8] A. Fröhlich and A. M. McEvett, Forms over rings with involution, Journal of Algebra 12 (1969), 79-104. MR 43:243

[9] - The representations of groups by automorphisms of forms, Journal of Algebra 12 (1969), 114-133. MR 39:1569

[10] Gordon James and Adalbert Kerber, The representation theory of the symmetric group, Encyclopedia of Mathematics and Its Applications, vol. 16, Addison-Wesley, Reading, MA, 1981. MR 83k:20003

[11] Max-Albert Knus, Alexander Merkurjev, Markus Rost, and Jean-Pierre Tignol, The book of involutions, Colloquium Publications, vol. 44, American Mathematical Society, Providence, R.I., 1998. MR 2000a:16031

[12] H. Koch, Number theory II, Encyclopaedia of Mathematical Sciences, vol. 62, Springer-Verlag, 1992. MR 98g:11118 (reprint)

[13] A. I. Malcev, On semisimple subgroups of Lie groups, Amer. Math. Soc. Transl. No. 33 (1950); reprint, Amer. Math. Soc. Transl. (1) 9 (1962), 172-213. MR 12:317c

[14] A. M. McEvett, Forms over semisimple algebras with involution, Journal of Algebra 12 (1969), 105-113. MR 43:244 
[15] John Milnor, On isometries of inner product spaces, Invent. Math. 8 (1969), 83-97. MR 40:2764

[16] Gabriele Nebe, Invariants of orthogonal G-modules from the character table, Experimental Mathematics 9 (2000), 623-629. CMP 2001:06

[17] _ Orthogonal Frobenius reciprocity, Journal of Algebra 225 (2000), 250-260. MR 2000m:20003

[18] Jürgen Neukirch, Algebraische Zahlentheorie, Springer-Verlag, Berlin, Heidelberg, 1992. MR 2000m:11104 (English transl.)

[19] O. T. O'Meara, Introduction to Quadratic Forms, Grund. d. math. Wiss., vol. 117, SpringerVerlag, New York, Heidelberg, Berlin, 1963. MR 27:2485

[20] I. Reiner, Maximal orders, L.M.S. Monographs, Academic Press, Inc., London, New York, San Francisco, 1975. MR 52:13910

[21] W. Scharlau, Quadratic and hermitian forms, Grund. Math. Wiss., vol. 270, Springer-Verlag, New York, Heidelberg, Berlin, 1985. MR 86k:11022

[22] J.-P. Serre, Linear representations of finite groups, Graduate Texts in Mathematics, vol. 42, Springer-Verlag, New York, Heidelberg, Berlin, 1977. MR 56:8675

[23] C. T. C. Wall, Classification of Hermitian Forms. VI. Group rings, Ann. of Math. (2) 103 (1976), 1-80. MR 55:5720

Deptartment of Mathematics and Statistics, McMaster University, Hamilton, OnTARIO, CANADA, L8S $4 \mathrm{~K} 1$

E-mail address: riehm@mcmaster.ca 